\title{
Influence of Amorphous Boron Grain Size, High Isostatic Pressure, Annealing Temperature, and Filling Density of Unreacted Material on Structure, Critical Parameters, $n$-Value, and Engineering Critical Current Density in $\mathrm{MgB}_{2}$ Wires
}

\author{
Daniel Gajda 1,* Andrzej J. Zaleski ${ }^{1}$ (D), Andrzej Morawski ${ }^{2}$, Małgorzata Małecka ${ }^{1}$, Mustafa Akdoğan $^{3}$, \\ Firat Karaboğa ${ }^{4}$, Doğan Avcı ${ }^{3}$ D, , Hakan Yetiş ${ }^{3}$, Ibrahim Belenli ${ }^{3}$ and Tomasz Czujko ${ }^{5, *(D)}$ \\ 1 Institute of Low Temperature and Structure Research PAS, Okolna 2, 50-422 Wroclaw, Poland; \\ a.zaleski@intibs.pl (A.J.Z.); m.malecka@int.pan.wroc.pl (M.M.) \\ 2 Institute of High Pressure Physics PAS, Sokolowska 29/37, 01-142 Warsaw, Poland; amor@unipress.waw.pl \\ 3 Department of Physics, Bolu Abant Izzet Baysal University, 14280 Bolu, Turkey; \\ akdogan_m@ibu.edu.tr (M.A.); davci.0209@gmail.com (D.A.); hknyetis@gmail.com (H.Y.); \\ belenli_i@ibu.edu.tr(I.B.) \\ check for \\ updates \\ Citation: Gajda, D.; Zaleski, A.J.; \\ Morawski, A.; Małecka, M.; Akdoğan, \\ 4 Mehmet Tanrıkulu Health Services Vocational School, Bolu Abant Izzet Baysal University, 14030 Bolu, Turkey; \\ karabogafirat@ibu.edu.tr \\ 5 Institute of Materials Science and Engineering, Military University of Technology, Kaliskiego 2, \\ 00-908 Warsaw, Poland \\ * Correspondence: d.gajda@intibs.pl (D.G.); tomasz.czujko@wat.edu.pl (T.C.); Tel.: +48-261-839-445 (T.C.)
} M.; Karaboğa, F.; Avcı, D.; Yetiş, H.; Belenli, I.; Czujko, T. Influence of Amorphous Boron Grain Size, High Isostatic Pressure, Annealing Temperature, and Filling Density of Unreacted Material on Structure, Critical Parameters, $N$-Value, and Engineering Critical Current Density in $\mathrm{Mgb}_{2}$ Wires. Materials 2021, 14 , 3600. https://doi.org/10.3390/ma 14133600

Academic Editors: Gianluca Ghigo and Daniele Torsello

Received: 15 May 2021

Accepted: 23 June 2021

Published: 28 June 2021

Publisher's Note: MDPI stays neutral with regard to jurisdictional claims in published maps and institutional affiliations.

Copyright: (c) 2021 by the authors. Licensee MDPI, Basel, Switzerland. This article is an open access article distributed under the terms and conditions of the Creative Commons Attribution (CC BY) license (https:// creativecommons.org/licenses/by/ $4.0 /)$.

\begin{abstract}
Our results show that a lower density of unreacted $\mathrm{Mg}+\mathrm{B}$ material during an $\mathrm{Mg}$ solidstate synthesis reaction leads to a significant reduction in the quantity of the superconducting phase and lowers the homogeneity of the superconducting material. It also significantly reduces the irreversible magnetic field $\left(B_{\text {irr }}\right)$, critical temperature $\left(T_{c}\right)$, upper magnetic field $\left(B_{c 2}\right)$, engineered critical current density $\left(\mathrm{J}_{\mathrm{ec}}\right)$, and $n$-value, despite high isostatic pressure (HIP) treatment and the use of nanoboron in the sample. Our measurements show that samples with large boron grains with an $8 \%$ higher density of unreacted $\mathrm{Mg}+\mathrm{B}$ material allow better critical parameters to be achieved. Studies have shown that the density of unreacted material has little effect on $\mathrm{B}_{\mathrm{irr}}, \mathrm{T}_{\mathrm{C}}, \mathrm{B}_{\mathrm{c} 2}, \mathrm{~J}_{\mathrm{ec}}$, and the $n$-value for an $\mathrm{Mg}$ liquid-state synthesis reaction. The results show that the critical parameters during an $\mathrm{Mg}$ liquid-state synthesis reaction depend mainly on grain size. Nanoboron grains allow for the highest $\mathrm{B}_{\mathrm{irr}}, \mathrm{T}_{\mathrm{c}}, \mathrm{B}_{\mathrm{c} 2}, \mathrm{~J}_{\mathrm{ec}}$, and $n$-values. Scanning electron microscopy (SEM) images taken from the longitudinal sections of the wires show that the samples annealed under low isostatic pressure have a highly heterogeneous structure. High isostatic pressure heat treatment greatly improves the homogeneity of $\mathrm{MgB}_{2}$.
\end{abstract}

Keywords: $\mathrm{MgB}_{2}$ superconducting wires; boron grain size; density of unreacted material; high isostatic pressure; critical current density

\section{Introduction}

Currently, $\mathrm{MgB}_{2}$ wires are the most promising material for superconducting coil applications due to their inexpensive components and ability to be cooled in liquid hydrogen, or by a cryocooler. The application of superconducting wires is dependent on several factors, e.g., engineering critical current density $\left(\mathrm{J}_{\mathrm{ec}}\right)$, irreversible magnetic field $\left(\mathrm{B}_{\text {irr }}\right)$, critical temperature $\left(T_{\mathcal{C}}\right), n$-value (the log-log slope of the voltage vs. electrical current curves during the transition of the superconducting material from a superconducting state to a normal state), and resistance in the normal state $\left(R_{n}\right)$. These parameters depend on the grain size and purity of magnesium and boron, heat treatment temperature, isostatic pressure, admixture, and wire sheath. Research has shown that nanoamorphous boron allows more connections between grains to be obtained, improves critical current density 
$\left(\mathrm{J}_{\mathrm{c}}\right)$, and accelerates the synthesis reaction [1-7]. However, large grains of amorphous boron $(1 \mu \mathrm{m})$ result in a smaller number of connections between the grains, reduce the critical current density, and slow down the synthesis reaction [1,6]. However, nanoamorphous boron is much more expensive than large-grain amorphous boron, leading to a higher price for $\mathrm{MgB}_{2}$ wires. In addition, $\mathrm{J}_{\mathrm{c}}$ also depends on the purity and type of boron [2,4]. Research has shown that high purity boron can significantly increase $\mathrm{J}_{\mathrm{c}}, \mathrm{B}_{\mathrm{irr}}$, and $\mathrm{T}_{\mathrm{c}}$. Moreover, experiments have shown that amorphous boron provides higher $\mathrm{J}_{\mathrm{C}}$ and $\mathrm{B}_{\mathrm{irr}}$ values than crystalline boron [1]. The research presented by Kim et al. indicates that nanocrystalline boron has a greater number of defects (e.g., dislocations, stresses) than large crystalline boron [1]. Additionally, these studies show that a greater number of structural defects in boron can accelerate the formation of the $\mathrm{MgB}_{2}$ superconducting phase.

Thermal treatment under high isostatic pressure (HIP) increases the density and homogeneity of $\mathrm{MgB}_{2}$ material, leads to more connections between grains, reduces the size of voids, forms structural defects (e.g., dislocations and strains), and creates smaller $\mathrm{MgB}_{2}$ grains [6,8-11]. Additionally, studies show that isostatic pressure increases the melting point of $\mathrm{Mg}[11,12]$, allowing synthesis reactions to be carried out in the solid-state of $\mathrm{Mg}$. Moreover, thermal treatment under high isostatic pressure enhances the irreversible magnetic field and critical current density $[6,13,14]$.

Currently, synthesis reactions for $\mathrm{MgB}_{2}$ materials are performed by two methods: $\mathrm{Mg}$ solid-state and $\mathrm{Mg}$ liquid-state. The reaction in the solid-state of $\mathrm{Mg}$ forms small $\mathrm{MgB}_{2}$ grains, smaller voids, and a greater number of intergrain connections [15-18]. The disadvantage of this reaction is the residual unreacted $\mathrm{Mg}$ and $\mathrm{B}$, which can be eliminated by applying a long annealing time [11]. Synthesis reactions in the liquid state of $\mathrm{Mg}$ can be conducted in a short time, allowing one to obtain a large number of superconducting phases after a short thermal treatment time. This type of reaction leads to the formation of large $\mathrm{MgB}_{2}$ grains, large voids, and fewer connections between grains, resulting in a degradation in critical current density $[15,18]$.

A study of the $\mathrm{MgB}_{2}$ material showed that the density of the unreacted material $(\mathrm{Mg}$ + 2B) affects the critical parameters of $\mathrm{MgB}_{2}$ wires. Flükiger et al. [19], Hossain et al. [20], and Akdoğan et al. [21] showed that a higher density of unreacted material $(\mathrm{Mg}+2 \mathrm{~B})$ increases critical current density in $\mathrm{MgB}_{2}$ wires. The core density of the unreacted $(\mathrm{Mg}+$ 2B) depends on the outer sheath material of the wires. Sheathing with a high hardness, e.g., $\mathrm{Fe}$, results in a higher unreacted core density than sheathing with a lower hardness, e.g., $\mathrm{Cu}$ [22]. Tanaka et al. showed that a low mass density of unreacted $\mathrm{Mg}+2 \mathrm{~B}$ in the wire leads to a reduction in $\mathrm{J}_{\mathrm{C}}$ for the superconducting coil [23]. Susner et al. indicated that both cold pressure and hot pressure processes increase the density of $\mathrm{MgB}_{2}$ material (after heat treatment) and improve the $\mathrm{J}_{\mathrm{C}}$ of $\mathrm{MgB}_{2}$ wires [24].

The application of $\mathrm{MgB}_{2}$ wires depends mainly on two factors: $n$-value and Jec. The $n$-value describes the transition from a superconducting state to a normal state [25-27] and depends on intrinsic (e.g., connections between grains, grain microstructure, and pinning centers) and extrinsic (e.g., filament distribution and quantity) factors. Kim et al. noted that a wire with a high $n$-value is used for a magnet to reduce its resistive component. Additionally, research shows that a high-quality sample has a high $n$-value. Moreover, Kim et al. indicated that $\mathrm{MgB}_{2}$ wires with a highly uniform microstructure reduce the dissipation for magnet applications [25]. Research by Motaman et al. also indicates that a more homogeneous microstructure causes a larger $n$-value, and that the $n$-value mostly decreases due to the inhomogeneous parts of samples [26]. Kim et al. showed that a small $n$-value, which leads to thermal and electrical dissipation, is characteristic of $\mathrm{MgB}_{2}$ superconductors $[25,26]$. Motaman et al. suggested that structural defects (dislocations) lead to an increase in $\mathrm{B}_{\mathrm{c} 2}$ and $\mathrm{J}_{\mathrm{c}}$ in high magnetic fields but reduce the $n$-value [26]. The results presented by Parizh et al. show that an $n$-value of 20 allows the use of only 50\% of the potential critical current in superconducting wires and tapes [27]. Motaman et al. indicated that superconducting wires are used in magnets when the $n$-value is in the range of 50 to 100 [26]. The $\mathrm{J}_{\mathrm{ec}}$ of the wires is used as a parameter in designing superconducting 
magnets. This value determines the critical current density $\left(\mathrm{J}_{\mathrm{c}}\right)$ for the cross-section of superconducting wire and tape (e.g., diffusion barrier, wire sheath, heat matrix, and superconducting material) [28,29]. Depending on the device in which the superconducting coils are used, the $\mathrm{J}_{\mathrm{ec}}$ value is currently $20-50 \mathrm{~A} / \mathrm{mm}^{2}$ for magnetic resonance imaging (MRI) magnets, $20-50 \mathrm{~A} / \mathrm{mm}^{2}$ for large coils for detectors, and $100-200 \mathrm{~A} / \mathrm{mm}^{2}$ for laboratory solenoids [28].

$B_{\text {irr }}$ is dependent on high-field pinning centers that are created by structural defects, e.g., dislocations, stresses, strains, and substitutions of $\mathrm{C}$ for $\mathrm{B}$ [29-32]. $\mathrm{MgB}_{2}$ material has an advantage of having a high critical temperature of $39 \mathrm{~K}$ [33]. Thus, cooling of the $\mathrm{MgB}_{2}$ wires and tapes can be achieved using liquid hydrogen, cryocoolers, or solidified nitrogen, which significantly reduces the running costs of superconducting devices. Additionally, $\mathrm{MgB}_{2}$ material has a low resistivity in the normal state of $0.38 \mu \Omega \mathrm{cm}$ [34]. This facilitates the design of protections against damage to the wires during the transition from a superconducting state to a normal state.

Our research aim was to show the effects of boron grain size, density of unreacted material, magnesium state (solid or liquid), and thermal treatment under high isostatic pressure on the formation of the $\mathrm{MgB}_{2}$ superconducting phase, the structure of the superconducting material, and critical parameters $\left(\mathrm{B}_{\mathrm{irr}}, \mathrm{B}_{\mathrm{c} 2}, \mathrm{~T}_{\mathrm{c}}, \mathrm{R}_{\mathrm{n}}\right.$, and $\left.\mathrm{J}_{\mathrm{c}}\right)$.

\section{Materials and Methods}

Monocore $\mathrm{MgB}_{2}$ wires with iron sheaths were produced using magnesium powder (purity: 99\% - particle sizes: 100-200 mesh 149-74 $\mu \mathrm{m}$ ) (Abant Izzet Baysal University, Bolu, Turkey) nd amorphous boron powder (Pavezyum Advanced Chemicals, İstanbul, Turkey).

Samples A to E were made using amorphous boron of $1 \mu \mathrm{m}$ particle size (Table 1). The samples from $\mathrm{F}$ to $\mathrm{J}$ were manufactured with amorphous boron mixtures: $50 \mathrm{wt} \%$ amorphous boron of $1 \mu \mathrm{m}$ particle size, and $50 \mathrm{wt} \%$-nanoamorphous boron of $0.25 \mu \mathrm{m}$ particle size (Table 1). Finally, the samples from $\mathrm{K}$ to $\mathrm{O}$ were fabricated with amorphous boron of $0.25 \mu \mathrm{m}$ particle size.

Table 1. Thermal treatment parameters and initial powder composition of the samples.

\begin{tabular}{cccccc}
\hline No. & $\begin{array}{c}\text { Annealing } \\
\text { Temperature } \\
{\left[{ }^{\circ} \mathrm{C}\right]}\end{array}$ & $\begin{array}{c}\text { Annealing } \\
\text { Time } \\
{[\mathrm{Min}]}\end{array}$ & $\begin{array}{c}\text { Isostatic } \\
\text { Pressure }\end{array}$ & $\begin{array}{c}\text { Boron Grain } \\
\text { Size }[\mathrm{mm}]\end{array}$ & $\begin{array}{c}\text { Density of the } \\
\text { Unreacted } \\
\text { Material } \\
{\left[\mathrm{G} / \mathrm{cm}^{3}\right]}\end{array}$ \\
\hline $\mathrm{A}$ & 680 & 40 & $0.1 \mathrm{MPa}$ & 1 & 1.5 \\
$\mathrm{~B}$ & 700 & 40 & $0.1 \mathrm{MPa}$ & 1 & 1.5 \\
$\mathrm{C}$ & 740 & 40 & $0.1 \mathrm{MPa}$ & 1 & 1.5 \\
$\mathrm{D}$ & 700 & 40 & $1.1 \mathrm{GPa}$ & 1 & 1.5 \\
$\mathrm{E}$ & 740 & 40 & $1.1 \mathrm{GPa}$ & 1 & 1.5 \\
$\mathrm{~F}$ & & & & $50 \mathrm{wt} \%-1$ & \\
& 680 & 40 & $0.1 \mathrm{MPa}$ & $50 \mathrm{wt} \%-0.25$ & 1.5 \\
$\mathrm{G}$ & 700 & 40 & $0.1 \mathrm{MPa}$ & $50 \mathrm{wt} \%-1$ & 1.5 \\
& & & & $50 \mathrm{wt} \%-1$ & \\
$\mathrm{H}$ & 740 & 40 & $0.1 \mathrm{MPa}$ & $50 \mathrm{wt} \%-0.25$ & 1.5 \\
& & & & $50 \%-1$ & \\
$\mathrm{I}$ & 700 & 40 & $1.1 \mathrm{GPa}$ & $50 \%-0.25$ & 1.5 \\
& & & & $50 \%-1$ & \\
$\mathrm{~J}$ & 740 & 40 & $1.1 \mathrm{GPa}$ & $50 \%-0.25$ & 1.5 \\
$\mathrm{~K}$ & 680 & 40 & $0.1 \mathrm{MPa}$ & 0.25 & 1.38 \\
$\mathrm{~L}$ & 700 & 40 & $0.1 \mathrm{MPa}$ & 0.25 & 1.38 \\
$\mathrm{M}$ & 740 & 40 & $0.1 \mathrm{MPa}$ & 0.25 & 1.38 \\
$\mathrm{~N}$ & 700 & 40 & $1.1 \mathrm{GPa}$ & 0.25 & 1.38 \\
$\mathrm{O}$ & 740 & 40 & $1.1 \mathrm{GPa}$ & 0.25 & 1.38 \\
\hline
\end{tabular}


The stoichiometric precursor $\mathrm{Mg}+2 \mathrm{~B}$ powder was mixed by ball milling for $3 \mathrm{~h}$ using a Pascall Engineering 1600 VSA ball mill at $150 \mathrm{rpm}$ and a ball-powder ratio of 4:1. The initial filling density of the filled powder for the samples from A to J was approximately $1.5 \mathrm{~g} / \mathrm{cm}^{3}$, and the filling density for the samples from $\mathrm{K}$ to $\mathrm{O}$ was approximately $1.38 \mathrm{~g} / \mathrm{cm}^{3}$ (Table 1) [21]. A cold drawing technique, with some intermediate heat treatment steps, was used to produce wire samples with a final diameter of $1.00-\mathrm{mm}$. Details of the production methods are presented in [21]. All samples were heated in an argon atmosphere at temperatures ranging from $680^{\circ} \mathrm{C}$ to $740{ }^{\circ} \mathrm{C}$, and under isostatic pressures ranging from $0.1 \mathrm{MPa}$ to $1.1 \mathrm{GPa}$ for $40 \mathrm{~min}$, as listed in Table 1 [35].

Measurements of $B_{i r r}, T_{c}, B_{c 2}$, and $R_{n}$ were made using a physical property measurement system (Physical Property Measurement System (PPMS), Model 7100, $15 \mathrm{~Hz}$ and AC current, $\mathrm{I}_{\mathrm{AC}}=100 \mathrm{~mA}$, Quantum Design, San Diego, CA, USA). The critical parameters $B_{\text {irr }}, T_{c}$, and $B_{c 2}$ were identified for the criteria of $10 \%, 50 \%$, and $90 \%$ resistance in the normal state [18]. The resistance in the normal state $\left(R_{n}\right)$ for $B=0$ T was determined at $40 \mathrm{~K}$. However, magnetoresistance was determined in the range of the magnetic field from $0 \mathrm{~T}$ to $14 \mathrm{~T}$. Transport critical current measurements were made using a measurement system with a bitter magnet up to $14 \mathrm{~T}$ at a temperature of $4.2 \mathrm{~K}$. The critical current $\left(\mathrm{I}_{\mathrm{c}}\right)$ was determined based on the $1 \mu \mathrm{m} / \mathrm{cm}$ criterion [29]. Jec was calculated from the relationship $\mathrm{J}_{\mathrm{ec}}=\mathrm{I}_{\mathrm{c}} / \mathrm{S}$ (S-wire cross-section) [29]. The $n$-values were determined from characteristic transport measurements [27]. The $\mathrm{J}_{\mathrm{ec}}$ and $n$-values for all samples were tested at the temperature of liquid helium (4.2 K).

The structure and composition studies were performed by using a scanning electron microscope (FEI Nova Nano SEM 230, Hillsboro, OR, USA) and Ultra Plus Scanning Electron Microscope (Zeiss, Oberkochen, Germany).

\section{Results and Discussion}

\subsection{Analysis of the Structure of $M g B_{2}$ Material}

The SEM images in Figure 1 show longitudinal sections of the samples from A to C, annealed in the temperature range from $680{ }^{\circ} \mathrm{C}$ to $740{ }^{\circ} \mathrm{C}$ under an isostatic pressure of $0.1 \mathrm{MPa}$. The longitudinal section allows for the best analysis of factors that significantly influence the engineering critical current density and $n$-value, e.g., connections between grains and homogeneity of the structure. The results indicate that the synthesis reaction in the sample from $\mathrm{A}$ to $\mathrm{C}$ is in the liquid state of $\mathrm{Mg}[9,10]$. Figure $1 \mathrm{a}, \mathrm{c}, \mathrm{e}$ shows that the layered structure is clustered in all samples (white arrow), creating dense superconducting regions with a highly heterogeneous distribution. The homogeneous layered structure was shown to significantly improve the connections between grains, leading to an increase in critical current density [18]. However, the heterogeneous distribution of dense regions reduces the connection between grains and leads to a reduction in $\mathrm{J}_{\mathrm{c}}$ and $\mathrm{J}_{\mathrm{ce}}$. Based on the results of Kim et al. [25] and Motaman et al. [26], we can conclude that the inhomogeneous distribution of dense regions results in a reduction in the $n$-value. Figure $1 \mathrm{~b}, \mathrm{~d}, \mathrm{f}$ shows that the $\mathrm{Mg}$ liquid-state synthesis reaction creates high-density regions, significantly improving the connection between grains in regions with a highly dense structure. Unfortunately, these dense regions are short in length, as seen in Figure 1.

Thermal treatment of the samples under a high isostatic pressure of $1.1 \mathrm{GPa}$ increases the melting point of magnesium up to $730^{\circ} \mathrm{C}$ [9], indicating that the synthesis reaction in sample D takes place when $\mathrm{Mg}$ is in the solid state. Figure 2a shows that thermal treatment under a high isostatic pressure of $1.1 \mathrm{GPa}$ and at an annealing temperature of $700{ }^{\circ} \mathrm{C}$ ensures a better homogeneity of the $\mathrm{MgB}_{2}$ material structure than sample $\mathrm{B}\left(70{ }^{\circ} \mathrm{C}\right.$ and $0.1 \mathrm{MPa})$. Sample D has more layers with smaller thickness and fewer large dense regions, which leads to more connections between the grains and improves the $n$-value. Figure $2 \mathrm{~b}$ shows that the layer structure in sample $\mathrm{D}\left(700^{\circ} \mathrm{C}\right.$ and $\left.1.1 \mathrm{GPa}\right)$ has a lower density than the layer structure of sample $\mathrm{B}\left(700^{\circ} \mathrm{C}\right.$ and $\left.0.1 \mathrm{MPa}\right)$. The relatively low layer density in sample $\mathrm{D}$ is due to the $\mathrm{Mg}$ solid-state synthesis reaction. $\mathrm{Mg}$ is in the liquid state in the thermal treatment of sample E $\left(740^{\circ} \mathrm{C}\right.$ and $\left.1.1 \mathrm{GPa}\right)$ [9]. Figure 2c shows that sample E 
$\left(740{ }^{\circ} \mathrm{C}\right.$ and $1.1 \mathrm{GPa}$ ) has more homogeneously distributed dense layers than sample $\mathrm{C}$ $\left(740{ }^{\circ} \mathrm{C}\right.$ and $\left.0.1 \mathrm{MPa}\right)$, allowing for more connections between grains, increasing $\mathrm{Jec}_{\mathrm{ec}}$, and improving the $n$-value.
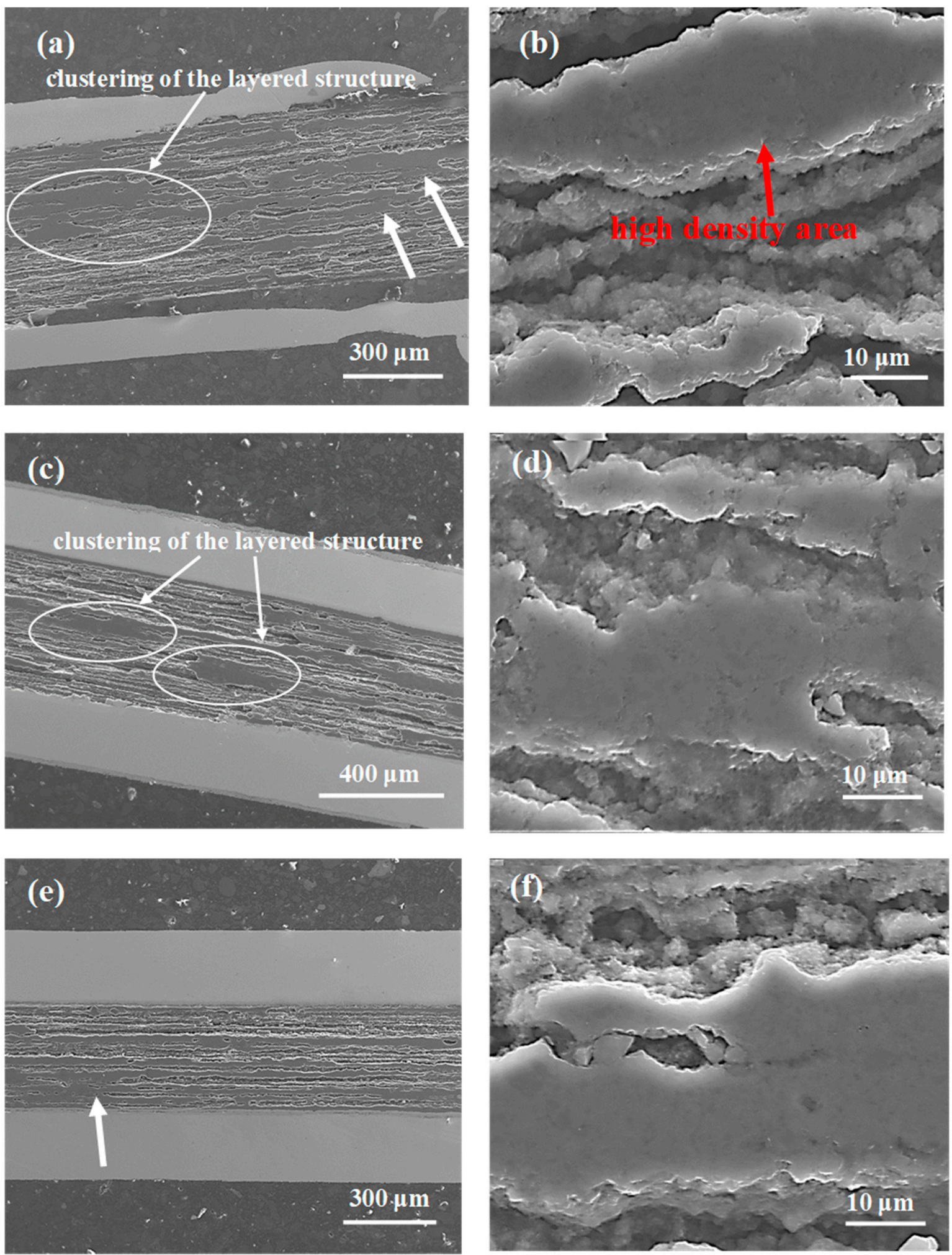

Figure 1. Longitudinal sections of $\mathrm{MgB}_{2}$ wires with boron grains of $1 \mu \mathrm{m}$ for isostatic pressures of $0.1 \mathrm{MPa}(\mathbf{a}, \mathbf{b})$ sample $\mathrm{A}-$ annealing temperature of $680^{\circ} \mathrm{C}$; (c,d) sample B-annealing temperature of $700{ }^{\circ} \mathrm{C} ;(\mathbf{e}, \mathbf{f})$ sample $\mathrm{C}$-annealing temperature of $740{ }^{\circ} \mathrm{C}$. The white arrow indicates high-density areas that are not uniformly distributed in the wire structure. 

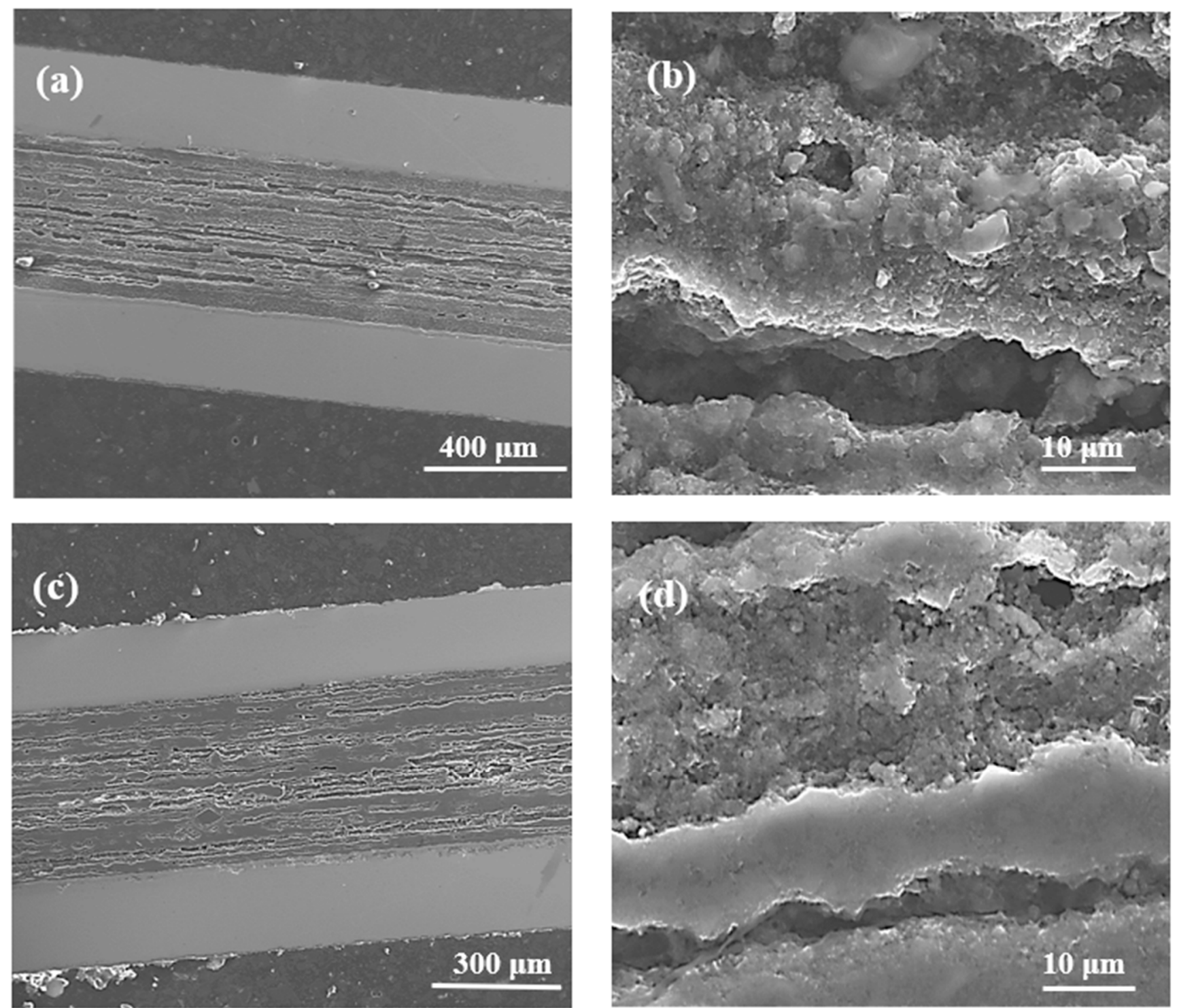

Figure 2. Longitudinal sections of $\mathrm{MgB}_{2}$ wires with boron grains of $1 \mu \mathrm{m}$ at an isostatic pressure of $1.1 \mathrm{GPa}(\mathbf{a}, \mathbf{b})$ sample $\mathrm{D}$-annealing temperature of $700{ }^{\circ} \mathrm{C} ;(\mathbf{c}, \mathbf{d})$ sample E-annealing temperature of $740{ }^{\circ} \mathrm{C}$.

Figure $2 \mathrm{~d}$ indicates that the density of the layered structure in sample $\mathrm{E}$ is similar to the density of the layers in sample C (Figure 1f). The SEM images taken from sample D $\left(700^{\circ} \mathrm{C}\right.$ and $\left.1.1 \mathrm{GPa}\right)$ and sample $\mathrm{E}\left(740^{\circ} \mathrm{C}\right.$ and $\left.1.1 \mathrm{GPa}\right)$ show that the $\mathrm{Mg}$ liquid synthesis reaction (Figure 2d) allows the formation of layers with a higher density than the $\mathrm{Mg}$ solid-state synthesis reaction (Figure 2b) under high isostatic pressure.

The SEM images in Figure 3 represent the longitudinal sections for samples $F$ to $G$ (a mixture of boron powder with grain sizes of $1 \mu \mathrm{m}$ and $0.25 \mu \mathrm{m}$ ). Figure $3 \mathrm{a}, \mathrm{b}$ shows that the mixture of boron forms a grain structure, with islands up to a size of $150 \mu \mathrm{m}$, at an annealing temperature of $680^{\circ} \mathrm{C}$. The formation of island structures may result from the deployment of small boron grains. Small boron grains react faster than large boron grains at a low temperature, indicating that the large boron forms longitudinal connections, while the small boron forms transverse connections, decreasing the homogeneity of the superconducting material. The results in Figure $3 \mathrm{c}-\mathrm{f}$ show that the annealing temperatures of $700{ }^{\circ} \mathrm{C}$ and $740{ }^{\circ} \mathrm{C}$ form a layered structure with inhomogeneous dense regions that reduce the number of connections between the grains. The results for samples $\mathrm{A}$ (boron$1 \mu \mathrm{m}$ ) and $\mathrm{F}$ (boron- $1 \mu \mathrm{m}$ and $0.25 \mu \mathrm{m}$ ) show that annealing at $680{ }^{\circ} \mathrm{C}$ creates different structures (sample A-layered structure and sample F-island structure), indicating that 
the $\mathrm{MgB}_{2}$ structure at low annealing temperature is dependent on particle size. The SEM images for samples $\mathrm{B}, \mathrm{C}, \mathrm{G}$, and $\mathrm{H}$ show that annealing temperatures of $700{ }^{\circ} \mathrm{C}$ and $740{ }^{\circ} \mathrm{C}$ result in the formation of a similar structure, indicating that the particle size at a high annealing temperature does not affect the structure in terms of the number and distribution of dense regions.
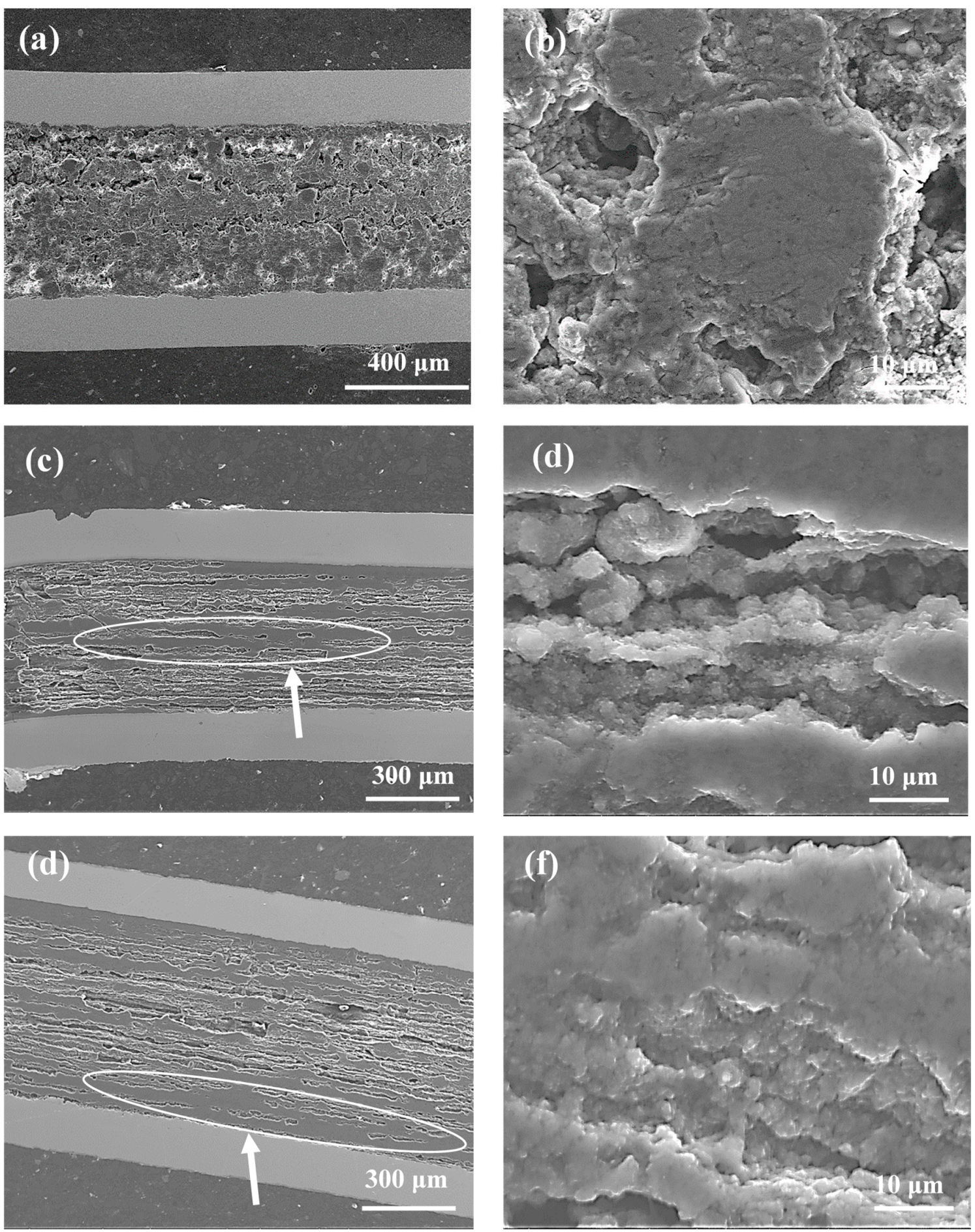

Figure 3. Longitudinal sections of $\mathrm{MgB}_{2}$ wires with boron grains of $1 \mu \mathrm{m}$ and $0.25 \mu \mathrm{m}$ for isostatic pressures of $0.1 \mathrm{MPa}(\mathbf{a}, \mathbf{b})$ sample F-annealing temperature of $680^{\circ} \mathrm{C} ;(\mathbf{c}, \mathbf{d})$ sample $\mathrm{G}$-annealing temperature of $700{ }^{\circ} \mathrm{C}$; (e,f) sample $\mathrm{H}$-annealing temperature of $740^{\circ} \mathrm{C}$. The white arrow indicates high-density areas that are not uniformly distributed in the wire structures. 
The synthesis reaction for sample I was performed as $\mathrm{Mg}$ was in the solid state [9]. Figure 4a shows that the thermal treatment under an isostatic pressure of $1.1 \mathrm{GPa}$ produces a greater number of dense regions with a better homogeneous distribution than sample $\mathrm{G}\left(700{ }^{\circ} \mathrm{C}\right.$ and $\left.0.1 \mathrm{MPa}\right)$. Both samples have dense regions similar to those in Figures $3 d$ and $4 b$. When the results in Figure $2 b$ are compared to those of Figure $4 b$, sample I $(1 \mu \mathrm{m}$ and $0.25 \mu \mathrm{m})$ can be seen to have a denser layered structure than sample D $(1 \mu \mathrm{m})$, indicating that the small boron grains and the high isostatic pressure accelerate the synthesis reaction when $\mathrm{Mg}$ is in the solid state.
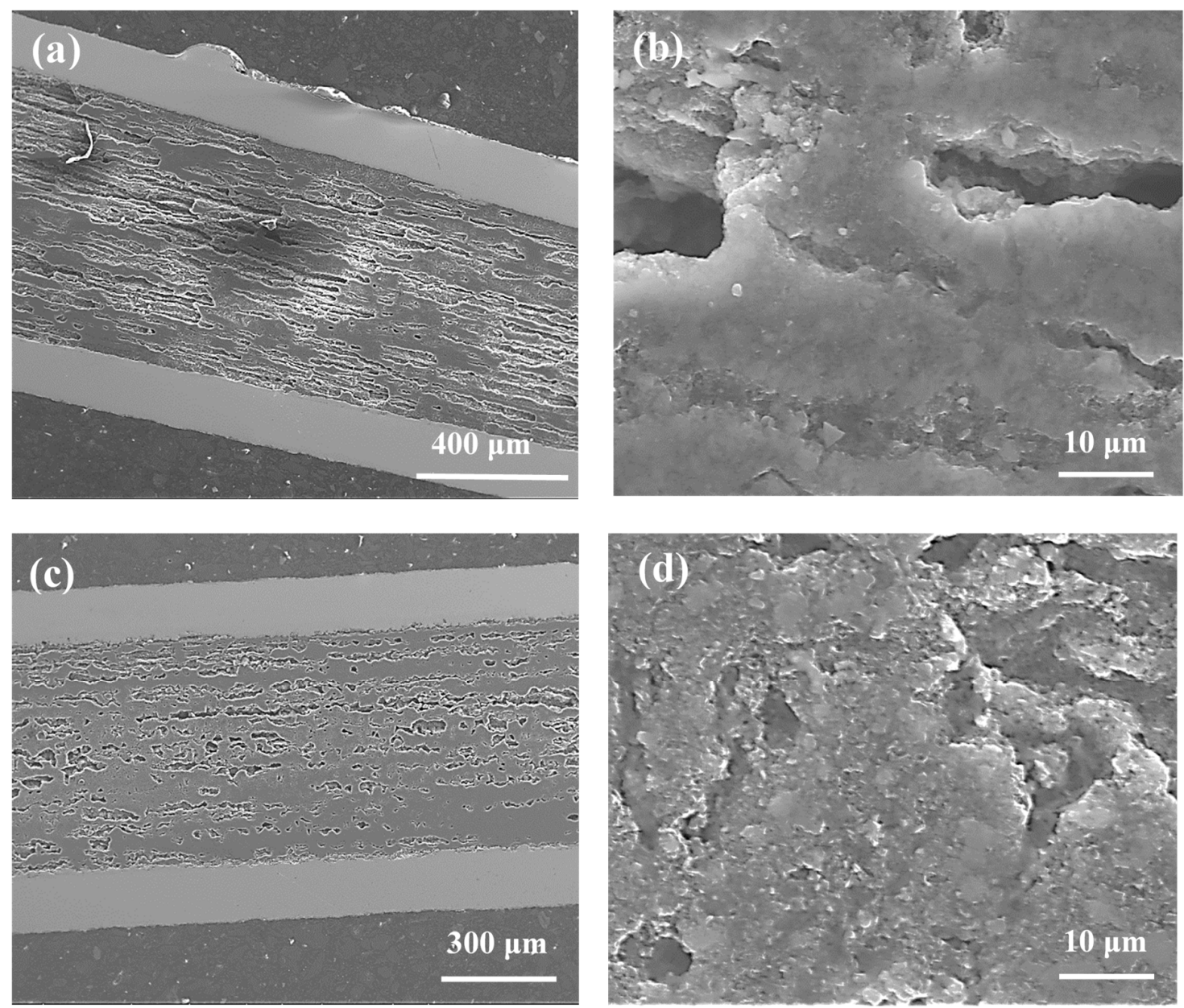

Figure 4. Longitudinal sections of $\mathrm{MgB}_{2}$ wires with boron grains of $1 \mu \mathrm{m}$ and $0.25 \mu \mathrm{m}$ for isostatic pressures of $1.1 \mathrm{GPa}$ (a,b) sample I-annealing temperature of $700^{\circ} \mathrm{C}$; (c,d) sample J-annealing temperature of $740^{\circ} \mathrm{C}$.

According to the results in [9], $\mathrm{Mg}$ is in the liquid state during the synthesis of sample $\mathrm{J}\left(740{ }^{\circ} \mathrm{C}\right.$ and $\left.1.1 \mathrm{GPa}\right)$. The results in Figures $3 \mathrm{~d}$ and $4 \mathrm{c}$ show that sample $\mathrm{J}$ has a layered structure with better homogeneity and denser regions than sample $\mathrm{H}\left(740^{\circ} \mathrm{C}\right.$ and $\left.0.1 \mathrm{MPa}\right)$, allowing the formation of a greater number of connections between the grains. From a comparison of the results in Figure 4a with those in Figure 4c, sample J can be seen to have a layered structure with a better homogeneity and denser regions than sample I. However, sample I (Figure $4 \mathrm{~b}$ ) has higher density layers than sample J (Figure 4d). The SEM images in Figures $2 c, d$ and $4 c, d$ show that high isostatic pressure and an annealing temperature of $740{ }^{\circ} \mathrm{C}$ contribute to the formation of a more homogeneous layered structure and a greater number of dense regions for the sample made of the boron mixture than the sample with boron grains $1 \mu \mathrm{m}$ in size.

The micrographs in Figure 5 for samples with nano boron show that thermal treatment at temperatures ranging from $680^{\circ} \mathrm{C}$ to $740{ }^{\circ} \mathrm{C}$ and under an isostatic pressure of $0.1 \mathrm{MPa}$ 
create a heterogeneous distribution of the layered structure and dense regions (white arrows). This process reduces the number of connections between the grains. Figure $5 b, d, f$ shows that increasing the annealing temperature from $700{ }^{\circ} \mathrm{C}$ to $740{ }^{\circ} \mathrm{C}$ for an isostatic pressure of $0.1 \mathrm{MPa}$ leads to a significant increase in the density of the layered structure, indicating that annealing at a higher temperature enhances the connection between the grains.
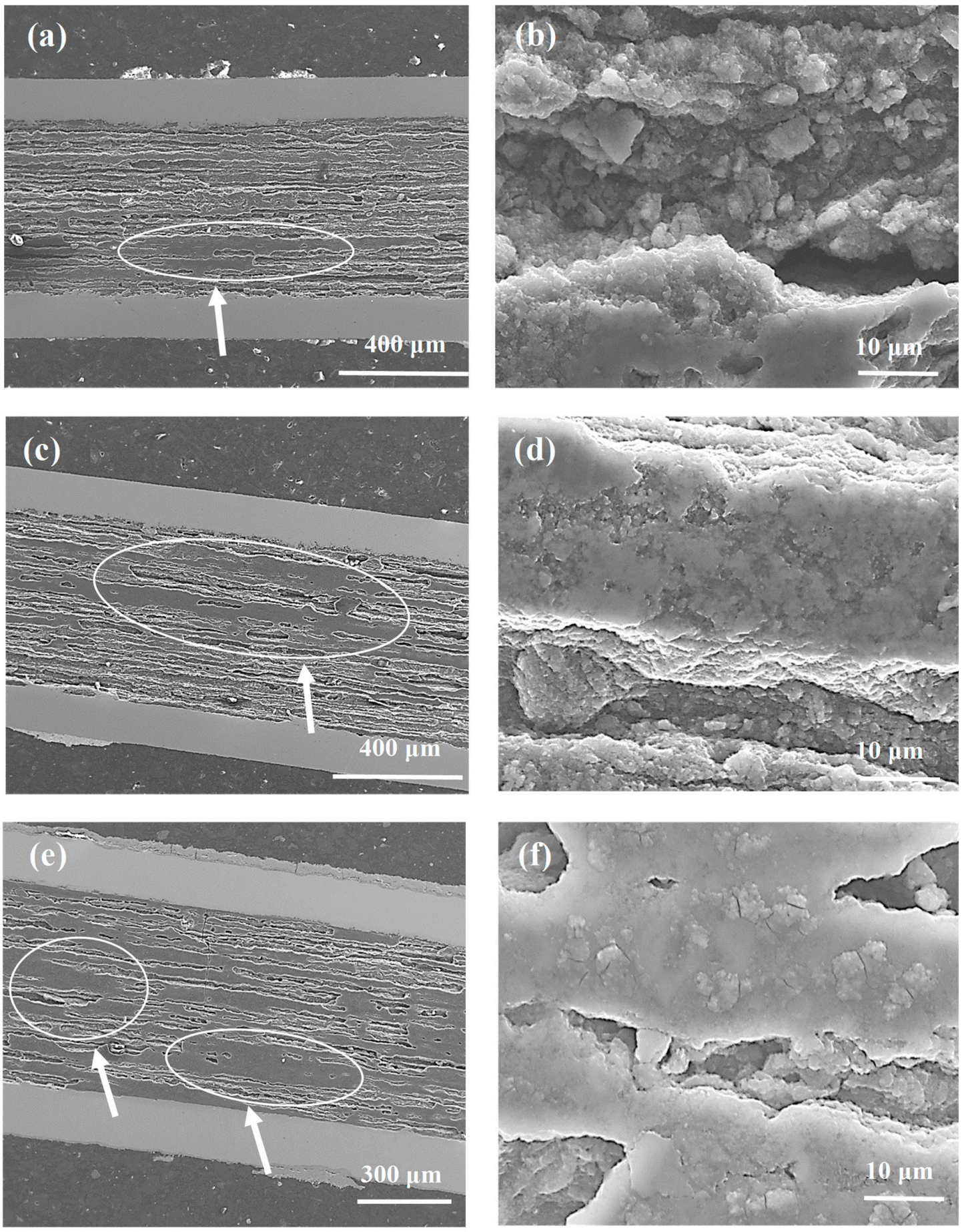

Figure 5. Longitudinal sections of $\mathrm{MgB}_{2}$ wires with boron grains of $0.25 \mu \mathrm{m}$ at an isostatic pressure of $0.1 \mathrm{MPa}(\mathbf{a}, \mathbf{b})$ sample $\mathrm{K}$-annealing temperature of $680^{\circ} \mathrm{C} ;(\mathbf{c}, \mathbf{d})$ sample L-annealing temperature of $700{ }^{\circ} \mathrm{C} ;(\mathbf{e}, \mathbf{f})$ sample $\mathrm{M}$-annealing temperature of $740^{\circ} \mathrm{C}$. The white arrow indicates high-density areas that are not uniformly distributed in the wire structures. 
The results in [9] show that the synthesis reaction in sample $\mathrm{N}$ is achieved for solidstate $\mathrm{Mg}$. The results obtained from the longitudinal section (Figure $6 \mathrm{a}-\mathrm{c}$ ) and the cross section (Figure 6d-backscattering analysis and EDX elemental maps (Figure 6e) show that sample $\mathrm{N}$ has a large number of unreacted regions (e.g., $\mathrm{B}, \mathrm{Mg}$ ) and a small amount of superconducting material as thin layers around the voids, leading to a reduction in homogeneity for sample $\mathrm{N}$ and decreasing the number of connections between the grains and the critical parameters. According to the results in [9], $\mathrm{Mg}$ is in the liquid state during the thermal treatment of sample $\mathrm{O}$ under high isostatic pressure. Figure $7 \mathrm{a}-\mathrm{c}$ (secondary electrons-SE) and Figure 7d (backscattering-(AsB)) show that a high annealing temperature and high isostatic pressure allow the formation of a large amount of superconducting material (no unreacted regions).

The SEM results from the longitudinal sections of the wires show that the core structures in Figures 1, 3, and 5 are very similar to each other, showing that the synthesis reaction in these samples is dependent mainly on the $\mathrm{Mg}$ state (solid/liquid) and less dependent on the density of unreacted material. The $\mathrm{E}, \mathrm{J}$, and $\mathrm{O}$ samples also look very similar, as seen in Figures $2 \mathrm{c}, \mathrm{d}, 4 \mathrm{c}, \mathrm{d}$, and $7 \mathrm{a}-\mathrm{c}$. Thermal treatment under high pressure was carried out in the liquid $\mathrm{Mg}$ state for these samples. High isostatic pressure improves the homogeneity of the $\mathrm{MgB}_{2}$ structure, indicating that the synthesis reaction in these samples is dependent mainly on the liquid state of $\mathrm{Mg}$ and high isostatic pressure, and weakly dependent upon the density of unreacted material.

\subsection{Analysis of Transport Measurements}

\subsubsection{Thermal Treatment under Low Isostatic Pressure}

Wang et al. [36] noted that the irreversible magnetic field is the maximum field in which $\mathrm{MgB}_{2}$ loses its ability to carry supercurrents. An irreversible magnetic field is also suggested to be able to increase by introducing effective pinning centers. Research shows that highfield pinning centers, such as dislocations, strains, and intragrain precipitations, effectively increase $B_{\text {irr }}$ [37]. The results in Figure 8 a for the A-C samples with large boron grains show that increasing the annealing temperature from $680^{\circ} \mathrm{C}$ to $700^{\circ} \mathrm{C}$ leads to an increase in $\mathrm{B}_{\text {irr }}$ by $10 \%$ at temperatures ranging from $4.2 \mathrm{~K}$ to $20 \mathrm{~K}$. An additional $40{ }^{\circ} \mathrm{C}$ increase in the annealing temperature results in a reduction of $B_{\text {irr }}$ by $20 \%$. Figure 1 shows that the $\mathrm{A}-\mathrm{C}$ samples have a similar layered structure and dense regions, indicating that increasing the annealing temperature is less effective in forming and accumulating high-field pinning centers for the sample with large boron grains. The transport measurements for the F-H samples (boron grains $-1 \mu \mathrm{m}$ and $0.25 \mu \mathrm{m}$ ) show that increasing the annealing temperature from $680^{\circ} \mathrm{C}$ to $700{ }^{\circ} \mathrm{C}$ leads to an increase in $\mathrm{B}_{\text {irr }}$ of $20 \%$ (Figure 8a). A further increase in the annealing temperature by $40^{\circ} \mathrm{C}$ does not increase $\mathrm{B}_{\text {irr. }}$. The annealing temperature of $700{ }^{\circ} \mathrm{C}$ seems to allow the formation of more effective high-field pinning centers. Additionally, the research reveals that $B_{\text {irr }}$ is strongly limited by the island structure (sample $\mathrm{F}$-Figure $3 b$ ). The results for $\mathrm{K}-\mathrm{O}$ samples show that increasing the annealing temperature from $680{ }^{\circ} \mathrm{C}$ to $740{ }^{\circ} \mathrm{C}$ does not change the $\mathrm{B}_{\text {irr }}$ value, suggesting that the formation and efficiency of high-field pinning are less dependent on the annealing temperature and might be more strongly dependent on nanoboron grains. The $\mathrm{B}_{\text {irr }}$ results indicate that nanosized boron grains increase $B_{\text {irr }}$ more efficiently (by $30 \%$ ) than large boron grains. Nanoboron forms much better high-field pinning centers than large boron for the liquid state of $\mathrm{Mg}$ during the synthesis reaction (despite the lower filling density of the unreacted material). 

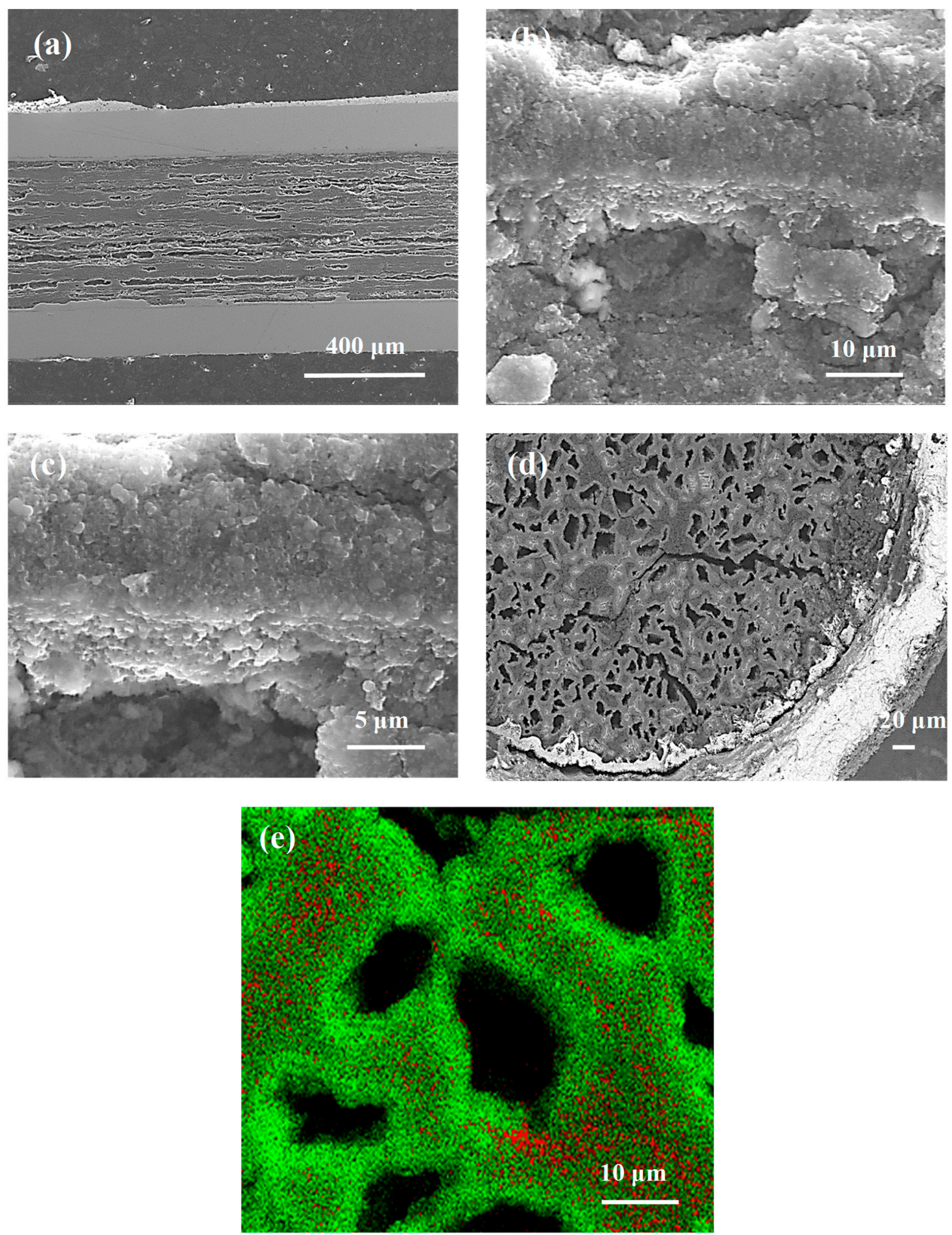

Figure 6. (a-c) The longitudinal section, (d) the cross-section-back scattering, and (e) the cross-section; EDX elemental maps for red-boron, green-magnesium for sample $\mathrm{N}$ (boron grains of $0.25 \mu \mathrm{m}$, isostatic pressure of $1.1 \mathrm{GPa}$, and annealing temperature of 700 . 

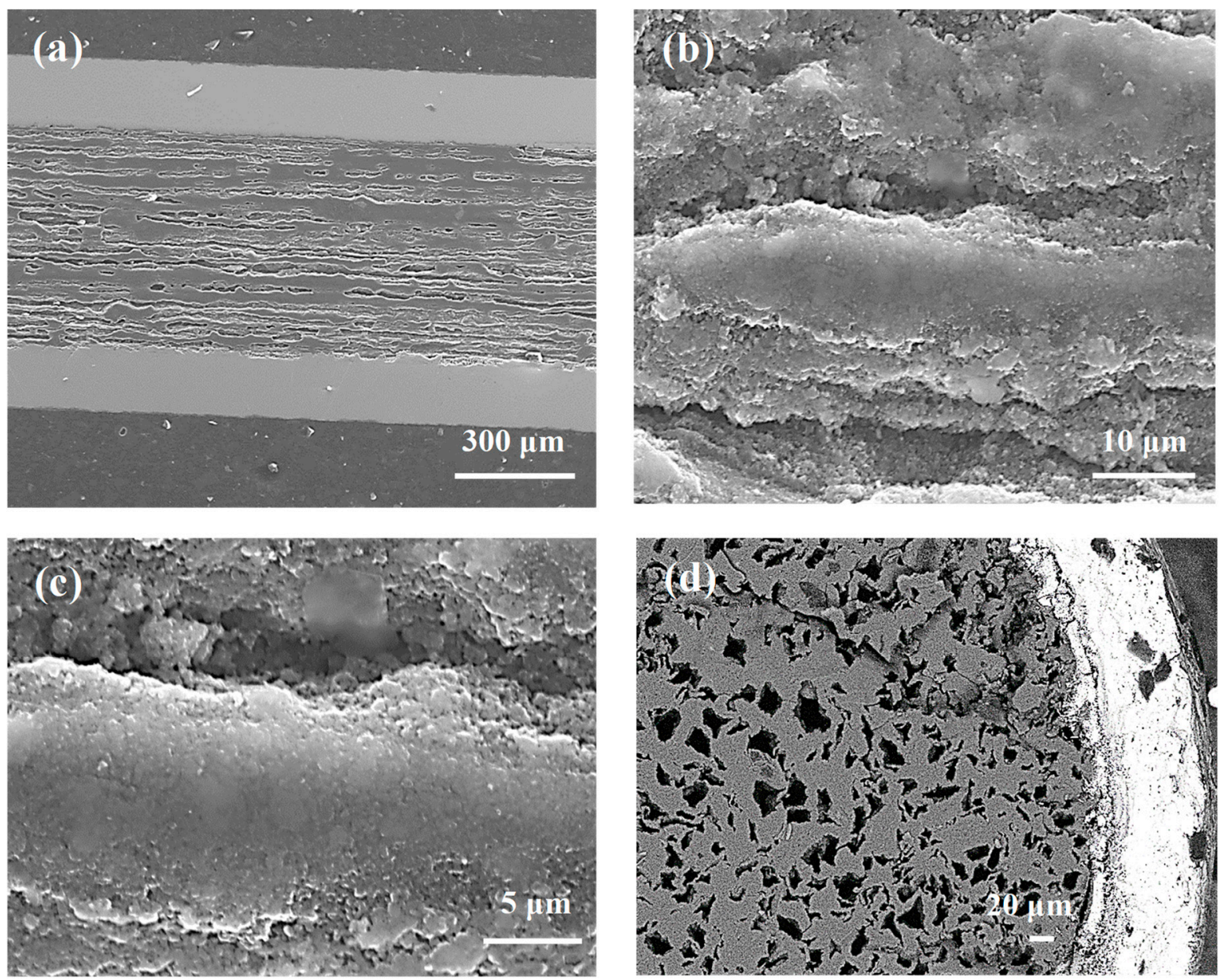

Figure 7. (a-c) The longitudinal section and (d) the cross section-backscattering analysis for sample $\mathrm{O}$ (boron grains of $0.25 \mu \mathrm{m}$ for isostatic pressure of $1.1 \mathrm{GPa}$ and annealing temperature of $740{ }^{\circ} \mathrm{C}$ ).
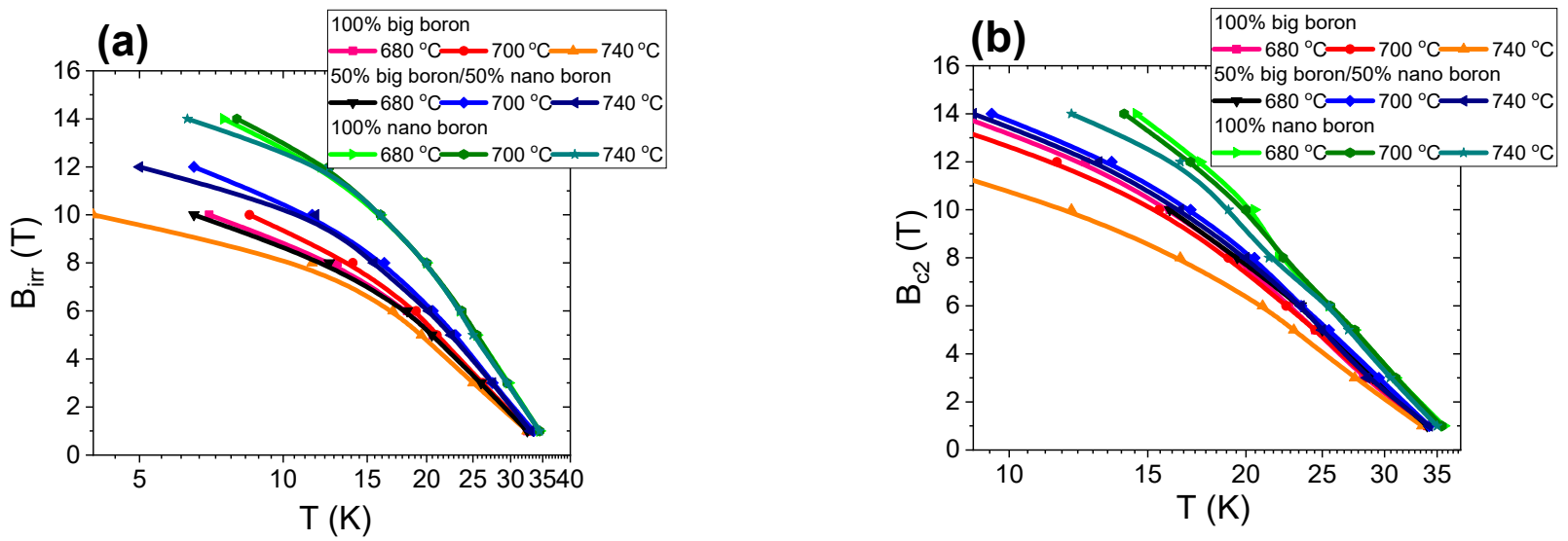

Figure 8. The temperature dependences of the irreversible magnetic field $\left(\mathrm{B}_{\mathrm{irr}}\right)(\mathbf{a})$ and the temperature dependences of the upper critical magnetic fields $\left(\mathrm{B}_{\mathrm{c} 2}\right)$ for an isostatic pressure of $0.1 \mathrm{MPa}(\mathbf{b})$.

Kazakov et al. [38] show that a decrease in superconducting coherence length leads to an increase in $\mathrm{B}_{\mathrm{c} 2}$. The superconducting coherence length decreases with structural defects, e.g., substitution of $C$ for $B$ in the $\mathrm{MgB}_{2}$ material $[38,39]$. The transport measurement results in Figure 8 b show that samples with a large boron have a similar $B_{\mathrm{c} 2}$. Only sample E (large grains) after heat treatment at $740{ }^{\circ} \mathrm{C}$ has a significantly lower $\mathrm{B}_{\mathrm{c} 2}$ by $20 \%$. These results 
indicate that increasing the annealing temperature in samples with large boron grains slightly increases the density of structural defects and slightly reduces the coherence length. The results in Figure $8 \mathrm{~b}$ for the sample with nanoboron show that increasing the annealing temperature from $700{ }^{\circ} \mathrm{C}$ to $740{ }^{\circ} \mathrm{C}$ does not change $\mathrm{B}_{\mathrm{c} 2}$, indicating that the annealing temperature slightly affects the formation of structural defects and the coherence length. When the transport measurement results for the samples with large boron and nanoboron are compared, nanoboron can be seen to significantly increase $B_{\mathrm{c} 2}$ by $25 \%$ (Figure $8 b$ ), revealing that nanoboron creates more structural defects than large boron. The results in Figure $8 a, b$ and the results in [15] show that the $B_{\text {irr }}$ and $B_{c 2}$ values of our wires are similar to the $B_{i r r}$ and $B_{c 2}$ values obtained for undoped $\mathrm{MgB}_{2}$ wires with nanoboron grains, suggesting that our $\mathrm{MgB}_{2}$ wires have high critical parameters.

The $T_{c}$ is dependent on several factors, e.g., lattice constants, annealing temperature, starting material, admixtures, and material purity [38,39]. Moreover, Buzea et al. reported that shrinkage of $\mathrm{MgB}_{2}$ material reduces the $\mathrm{T}_{\mathrm{c}}$ by $1 \mathrm{~K}$ [40]. The results in Figure 9 show that increasing the annealing temperature from $680{ }^{\circ} \mathrm{C}$ to $740{ }^{\circ} \mathrm{C}$ slightly affects the $T_{c}$ value. In addition, the results in Figure 9 show that the samples made with only nanoboron have a $\mathrm{T}_{\mathrm{c}}$ of $0.8 \mathrm{~K}$ higher than the samples made with large boron and boron mixtures $(1 \mu \mathrm{m}$ and $0.25 \mu \mathrm{m})$. These results show that the grain size of boron has a more significant influence on $T_{c}$ than the annealing temperature. When comparing our results in Figure 9 with the results in [15], we see that the $\mathrm{T}_{\mathrm{c}}$ of our $\mathrm{MgB}_{2}$ wires with nanoboron grains is approximately $1.5 \mathrm{~K}$ higher than the $\mathrm{T}_{\mathrm{c}}$ of undoped $\mathrm{MgB}_{2}$ wires with nanoboron grains [15].

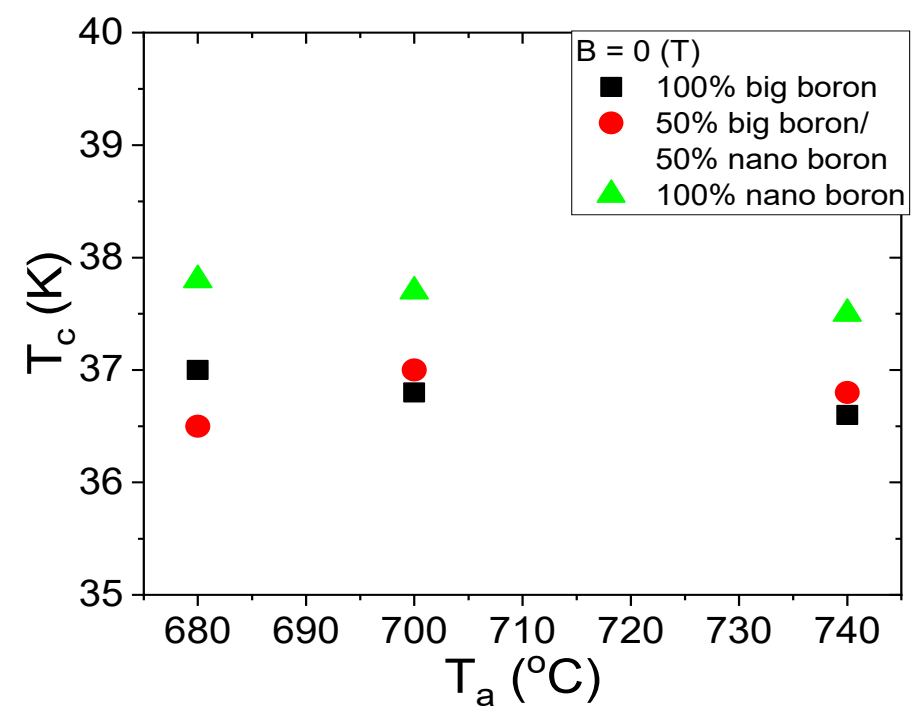

Figure 9. The annealing temperature $\left(T_{a}\right)$ dependences of the critical temperature $\left(T_{c}\right)$ for an isostatic pressure of $0.1 \mathrm{MPa}$ and $\mathrm{B}=0 \mathrm{~T}$.

The results in Figure 10a show that an annealing temperature of $740{ }^{\circ} \mathrm{C}$ results in a $15 \%$ increase in normal state resistance $\left(R_{n}\right)$ when the magnetic field is increased from $0 \mathrm{~T}$ to $14 \mathrm{~T}$. In the $\mathrm{A}$ and $\mathrm{B}$ samples heated at $680{ }^{\circ} \mathrm{C}$ and $700{ }^{\circ} \mathrm{C}$, no increase was observed in normal state resistance as the magnetic field increased because sample $\mathrm{C}$ was annealed at a higher temperature, indicating that magnetoresistance occurs due to the $\mathrm{Mg}$ and $\mathrm{B}$ compounds containing Fe and does not depend on unreacted Mg. Magnetoresistance caused by unreacted Mg was not seen even in samples A and B that were annealed at a lower temperature. The transport measurements in the zero magnetic field show that the $\mathrm{K}-\mathrm{L}$ samples have lower resistance ( $30 \%$ less) in the normal state than the $\mathrm{A}-\mathrm{C}$ and $\mathrm{F}-\mathrm{H}$ samples, indicating that nanoboron creates a larger number of connections between the grains than large boron and a mixture of boron. 


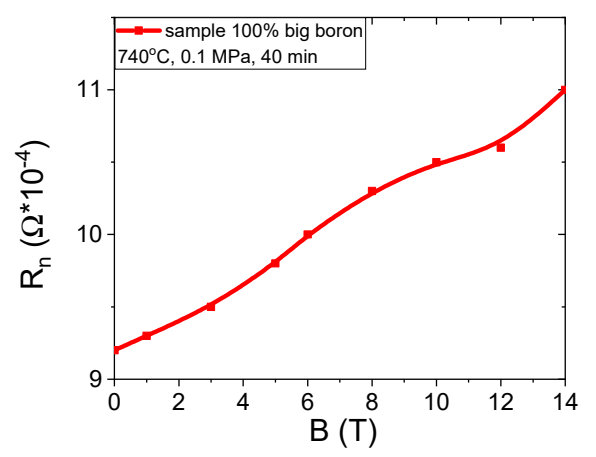

Figure 10. The magnetic field (B) dependences of the normal state resistance for sample C.

The results in Figure 11 for samples A-C (grain size of $1 \mu \mathrm{m}$ ) show that increasing the annealing temperature from $680^{\circ} \mathrm{C}$ to $740{ }^{\circ} \mathrm{C}$ significantly increases the $\mathrm{Jec}_{\mathrm{ec}}$ by approximately $50 \%$ in the magnetic field range from $3 \mathrm{~T}$ to $6 \mathrm{~T}$. The high annealing temperature in $\mathrm{MgB}_{2}$ wires with large boron grains seems to form mainly on surface pinning centers [29]. $\mathrm{J}_{\mathrm{ec}}$ was determined with the ratio $\mathrm{J}_{\mathrm{ec}}=\mathrm{I}_{\mathrm{c}} / \mathrm{S}$ (S-wire cross-section) [29]. The transport results for sample F-H (mixture of boron grains $-1 \mu \mathrm{m}$ and $0.25 \mu \mathrm{m}$ ) show that increasing the annealing temperature from $680^{\circ} \mathrm{C}$ to $700{ }^{\circ} \mathrm{C}$ increases Jec by one order of magnitude (Figure 11). A further increase in annealing temperature leads to a slight reduction in $\mathrm{Jec}_{\mathrm{ec}}$. An annealing temperature of $700{ }^{\circ} \mathrm{C}$ for samples with a mixture of boron grains significantly increases the density of all types of pinning centers [29,37]. The results for the $\mathrm{K}-\mathrm{M}$ samples (nanoboron- $-0.25 \mu \mathrm{m}$ ) show that increasing the annealing temperature from $680{ }^{\circ} \mathrm{C}$ to $740{ }^{\circ} \mathrm{C}$ significantly increases $\mathrm{Jec}_{\mathrm{ec}}$ by $60 \%$ in the magnetic field range from $6 \mathrm{~T}$ to $8 \mathrm{~T}$ and significantly reduces Jec by $40 \%$ in magnetic fields above $8 \mathrm{~T}$ (Figure 11). These results indicate that the high annealing temperature in $\mathrm{MgB}_{2}$ wires with nanoboron mainly forms surface pinning centers. However, a lower annealing temperature in the sample with nanoboron more effectively creates high-field pinning centers. Based on the results in Figure 11, the mixture of boron grains $(1 \mu \mathrm{m}$ and $0.25 \mu \mathrm{m})$ can be considered to significantly increase the $\mathrm{J}_{\mathrm{ec}}$ at all annealing temperatures in comparison to the sample with large boron grains $(1 \mu \mathrm{m})$ because the addition of nanoboron increases the density of all types of pinning center [29,37]. A comparison of the results for samples with the boron mixture with the results for samples with nanoboron reveals that only nanoboron allows for high $\mathrm{J}_{\mathrm{ec}}$ in medium and high magnetic fields, and it meets the applicability requirements (e.g., $100 \mathrm{~A} / \mathrm{mm}^{2}, 50 \mathrm{~A} / \mathrm{mm}^{2}$, and $20 \mathrm{~A} / \mathrm{mm}^{2}$ ). Currently, studies show that the highest $\mathrm{J}_{\mathrm{ec}}$ is obtained in $\mathrm{MgB}_{2}$ wires made by internal $\mathrm{Mg}$ diffusion (IMD) [41,42]. Our sample $\mathrm{M}$ (nanoboron $-740{ }^{\circ} \mathrm{C}$ and $0.1 \mathrm{MPa}$ ) has $\mathrm{Jec}_{\mathrm{ec}} 100 \mathrm{~A} / \mathrm{mm}^{2}$ at $7 \mathrm{~T}$. This $\mathrm{Jec}_{\mathrm{ec}}$ is similar to the $\mathrm{J}_{\mathrm{ec}}$ of doped $\mathrm{MgB}_{2}$ wire (PIT) [43] and higher than the $\mathrm{J}_{\mathrm{ec}}$ of undoped $\mathrm{MgB}_{2}$ IMD wire (IMD) [44].

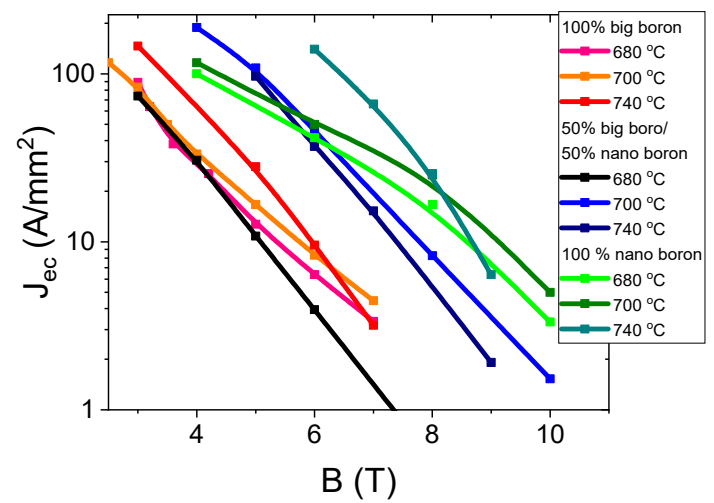

Figure 11. Transport $\mathrm{Jec}_{\mathrm{ec}}-\mathrm{B}$ curves for undoped $\mathrm{MgB}_{2}$ wires in a perpendicular magnetic field at an isostatic pressure of $0.1 \mathrm{MPa}$ at $4.2 \mathrm{~K}$. 
The $n$-value determines Jec, which can be used in superconducting coils [27]. The $n$-value was calculated using the formula in [45]. Our results in Figure 12 represent the relationships between the $n$-value, magnetic field, and engineering critical current density. This analysis helps identify which $\mathrm{MgB}_{2}$ wire can be used to build low-, middle- and high-field superconducting coils. The results in Figure 12a for an annealing temperature of $680{ }^{\circ} \mathrm{C}$ and isostatic pressure of $0.1 \mathrm{MPa}$ indicate that nanoboron grains allow the highest $n$-value, depending on B and Jec. The SEM images in Figure 5a show that the sample with nanoboron grains has a more homogeneous structure (e.g., layers have a lower thickness and fewer clustered layers) than a sample made with large boron grains (Figure 1a), leading to an increase in the number of connections between grains and a high $n$-value and Jec. The research shows that the lowest $n$-value belongs to the sample made with the mixture of boron grains $(1 \mu \mathrm{m}$ and $0.25 \mu \mathrm{m})$, indicating that the island structure most reduces the $n$-value and $\mathrm{Jec}_{\mathrm{ec}}$. Figure 3 a shows that sample $\mathrm{F}$ has the least homogeneous structure. Parizh et al. [27] indicated that an $n$-value of 20 allows the use of only $50 \%$ of the Jec value. The results for the annealing temperature of $680{ }^{\circ} \mathrm{C}$ indicate that $\mathrm{MgB}_{2}$ wires with large boron grains can be used for the construction of low-field superconducting coils (for $\mathrm{Jec}_{\mathrm{ec}}-40 \mathrm{~A} / \mathrm{mm}^{2}$ ). However, $\mathrm{MgB}_{2}$ wires with nanoborons can be used to build medium-field superconducting coils.

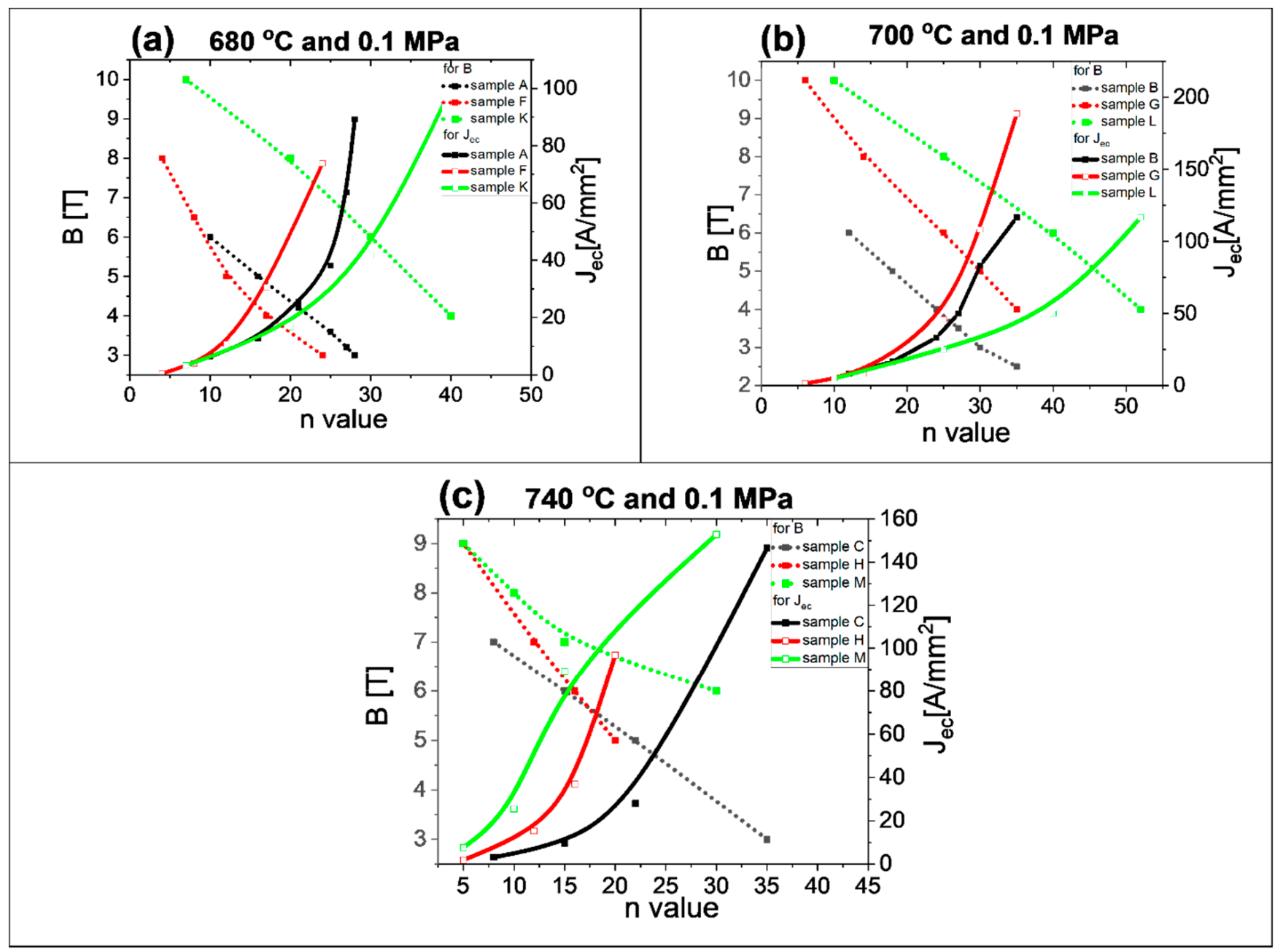

Figure 12. The dependence of the $n$-value on the magnetic field (B) perpendicular to the wire axis in undoped $\mathrm{MgB}_{2}$ wires for an isostatic pressure of $0.1 \mathrm{MPa}$ : (a) annealing temperature of $680^{\circ} \mathrm{C}$; (b) annealing temperature of $700{ }^{\circ} \mathrm{C}$; (c) annealing temperature of $740{ }^{\circ} \mathrm{C}$. 
The transport results after annealing at $700{ }^{\circ} \mathrm{C}$ and isostatic pressure of $0.1 \mathrm{MPa}$ (Figure 12b) show that the $\mathrm{MgB}_{2}$ wire with nanoboron grains has a higher $n$-value than the wires with large boron grains and a mixture of boron grains, e.g., $n$-value of 40 in $6 \mathrm{~T}$ for nanoboron, $n$-value of 12 in $6 \mathrm{~T}$ for large boron. The research also shows that the addition of nanoboron grains (sample G) also increases the $n$-value (Figure 12b). The SEM images in Figures $1 c, 3 c$, and $5 c$ show that the sample with nanoboron grains has a structure with thinner layers and fewer clustered layers than the sample with large and mixed boron grains, also leading to an improvement in $n$-value. The results in Figure $12 \mathrm{~b}$ show that an annealing temperature of $700{ }^{\circ} \mathrm{C}$ allows fabrication of only low-field superconducting coils from $\mathrm{MgB}_{2}$ wires with large boron grains. However, research shows that $\mathrm{MgB}_{2}$ wires with nanoboron grains, after annealing at $700{ }^{\circ} \mathrm{C}$, can be used to build high-field superconducting coils (up to $7.5 \mathrm{~T}$ ).

The transport measurements in Figure 12c show that the annealing temperature of $740{ }^{\circ} \mathrm{C}$ and isostatic pressure significantly increase the $n$-value in nanoboron grain $\mathrm{MgB}_{2}$ wires only under low and medium magnetic fields and slightly increase the $n$-value under high magnetic fields compared to the $\mathrm{MgB}_{2}$ wire produced from mixtures of boron grains.

A higher annealing temperature improves the homogeneity of the structure in $\mathrm{MgB}_{2}$ wires for large boron and a mixture of boron (Figures 1e and 3e), leading to an improvement in the n-value. However, the annealing temperature of $740{ }^{\circ} \mathrm{C}$ reduces the homogeneity of $\mathrm{MgB}_{2}$ wire with nanoboron (Figure 5e). The results of Figure $12 \mathrm{c}$ show that $\mathrm{MgB}_{2}$ wires with large boron and a mixture of boron can be used to build medium field superconducting coils (up to $5 \mathrm{~T}$ ).

The increase in the annealing temperature from $680{ }^{\circ} \mathrm{C}$ to $740{ }^{\circ} \mathrm{C}$ for $\mathrm{MgB}_{2}$ wires with large boron and a mixture of boron leads to an increase in the $n$-value. Additionally, the measurements show that increasing the annealing temperature from $680{ }^{\circ} \mathrm{C}$ to $740{ }^{\circ} \mathrm{C}$ for samples with nanoboron grains increases the $n$-value in low and medium magnetic fields and reduces the $n$-value under high magnetic fields, indicating that the $n$-value is related to $J_{\mathrm{ec}}$ and pinning centers because sample $\mathrm{M}$ after heat treatment at $740{ }^{\circ} \mathrm{C}$ has the highest $\mathrm{J}_{\mathrm{ec}}$ in low and medium magnetic fields and a significantly lower $\mathrm{J}_{\mathrm{ec}}$ in high magnetic fields than sample $\mathrm{L}\left(700^{\circ} \mathrm{C}\right)$. We obtained an $n$-value of 30 at $7.5 \mathrm{~T}$ for the $\mathrm{MgB}_{2}$ wire with nanoboron grains $\left(700^{\circ} \mathrm{C}\right.$ and $\left.0.1 \mathrm{MPa}\right)$. Li et al. [43] found that the $n$-value was 30 under $6 \mathrm{~T}$ for undoped $\mathrm{MgB}_{2}$ wires made by conventional continuous tube forming/filling (CTFF). Moreover, research in [25] showed that undoped $\mathrm{MgB}_{2}$ wires made by the IMD technique had an $n$-value of 30 under $8 \mathrm{~T}$, indicating that our undoped $\mathrm{MgB}_{2}$ wires have $\mathrm{J}_{\mathrm{ec}}$ and $n$-values similar to $\mathrm{Jec}_{\mathrm{ec}}$ and $n$-values of the best undoped $\mathrm{MgB}_{2}$ wires.

Our research shows that nanoboron after a reaction with $\mathrm{Mg}$ in the liquid state has a great influence on critical parameters $\left(\mathrm{B}_{\mathrm{irr}}, \mathrm{B}_{\mathrm{c} 2}, \mathrm{~T}_{\mathrm{c}}, \mathrm{J}_{\mathrm{ec}}\right.$, and $n$-value). In addition, we proved that the homogeneous structure of $\mathrm{MgB}_{2}$ material, e.g., uniform distribution of voids, thickness layers, and material density of $\mathrm{MgB}_{2}$, significantly affected the Jec and $n$ value in all samples (large boron, boron mixture, and small boron).

\subsubsection{Thermal Treatment at $700{ }^{\circ} \mathrm{C}$ under High Isostatic Pressure}

The results presented in [9] show that the melting point of $\mathrm{Mg}$ is approximately $730{ }^{\circ} \mathrm{C}$ for an isostatic pressure of $1.1 \mathrm{GPa}$. Thus, the synthesis reactions in samples $\mathrm{D}, \mathrm{I}$, and $\mathrm{N}$ are in the solid state of $\mathrm{Mg}$. However, $\mathrm{Mg}$ was in the liquid state for the samples B, G and $\mathrm{L}$ thermally treated at a low isostatic pressure of $0.1 \mathrm{MPa}$. The transport results for large boron grains $(1 \mu \mathrm{m})$ show that thermal treatment under high isostatic pressure does not improve $B_{\text {irr }}$ (Figure 13a), indicating that the $1.1 \mathrm{GPa}$ isostatic pressure does not improve or increase the density of high-field pinning centers in $\mathrm{MgB}_{2}$ wires with large boron grains for $\mathrm{Mg}$ solid-state synthesis reactions. 
(a)

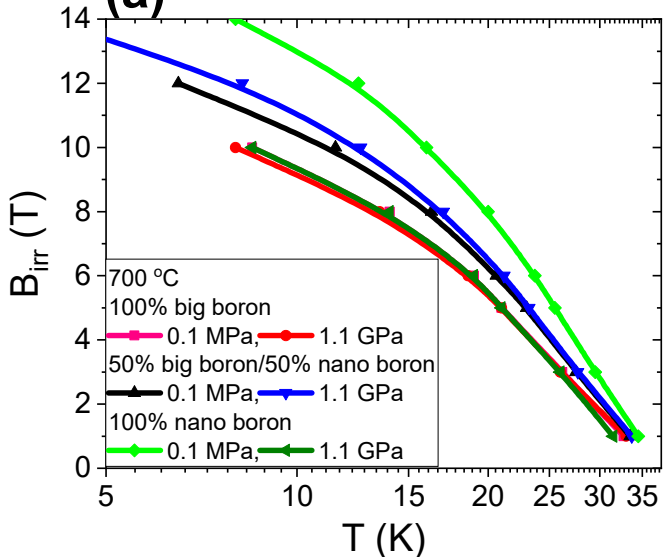

(b)

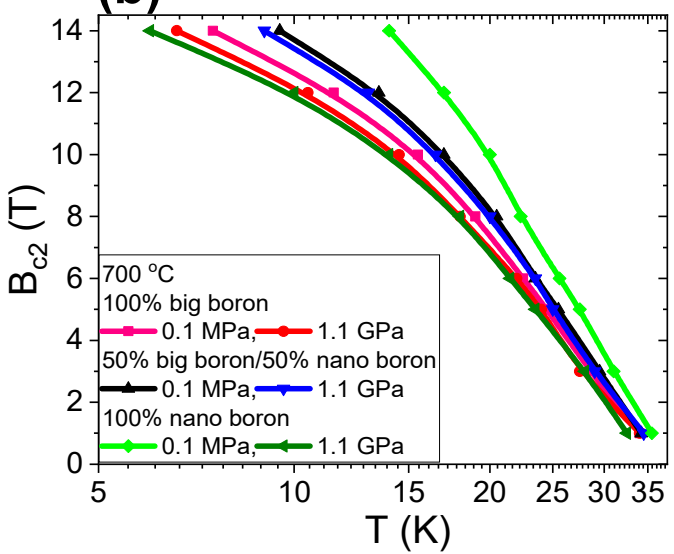

Figure 13. Temperature dependence of the irreversible magnetic field $\left(\mathrm{B}_{\mathrm{irr}}\right)(\mathbf{a})$ and of the upper magnetic field $\left(\mathrm{B}_{\mathrm{c} 2}\right)$ for an annealing temperature of $700{ }^{\circ} \mathrm{C}(\mathbf{b})$.

Further studies show that high isostatic pressure annealing for sample I with the boron mixture $(1 \mu \mathrm{m}$ and $0.25 \mu \mathrm{m})$ slightly increases $B_{\text {irr }}$ (Figure 13a), indicating that the isostatic pressure of $1.1 \mathrm{GPa}$ weakly improves the density of high-field pinning centers in $\mathrm{MgB}_{2}$ wires with the boron mixture when $\mathrm{Mg}$ is in the solid state. Transport measurements for sample $\mathrm{N}$ with nanoboron grains $(0.25 \mu \mathrm{m})$ show that thermal treatment under high isostatic pressure significantly reduces $B_{\text {irr }}$ by $30 \%$ (Figure 13a). The SEM results in Figure 6 show that the significant reduction in $B_{\text {irr }}$ is associated with a small superconducting phase and the large amount of unreacted $\mathrm{B}$ and $\mathrm{Mg}$ in sample $\mathrm{N}$. These results show that thermal treatment under high isostatic pressure for the solid state of $\mathrm{Mg}$ weakly forms high-field pinning centers in $\mathrm{MgB}_{2}$ wires with a lower unreacted material density. The research shows that the mixture of boron grains and HIP process increases $B_{\text {irr }}$ by $15 \%$ compared to large boron grains for the $\mathrm{Mg}$ solid-state synthesis reaction and the same density of unreacted material $\left(1.5 \mathrm{~g} / \mathrm{cm}^{3}\right)$. Studies show that nanoboron grains and the HIP process do not increase $\mathrm{B}_{\text {irr }}$ in comparison to large boron grains for the $\mathrm{Mg}$ solid-state synthesis reaction and the $8 \%$ lower filling density of unreacted material $\left(1.38 \mathrm{~g} / \mathrm{cm}^{3}\right)$. These results indicate that the synthesis reaction of $\mathrm{MgB}_{2}$ material in the solid state of $\mathrm{Mg}$ is more dependent on the density of unreacted material than the particle size of the boron. The transport measurements in Figure 13b show that heat treatment under high isostatic pressure slightly increases $B_{\mathrm{c} 2}$ in samples with large boron grains and a mixture of boron grains, indicating that the isostatic pressure of $1.1 \mathrm{GPa}$ weakly increases the density of the structural defects, which leads to a reduction in the coherence length. Studies for nanoboron grains (sample $\mathrm{N}$ ) show that high isostatic pressure annealing leads to a significant reduction in $\mathrm{B}_{\mathrm{c} 2}$ by $30 \%$ (Figure 13b). This significant reduction in Bc2 is the result large amount of unreacted $\mathrm{B}$ and $\mathrm{Mg}$, revealing that $\mathrm{Bc} 2$ is dependent on the density of the unreacted material for the $\mathrm{Mg}$ solid-state synthesis reaction and less dependent on boron grain size. However, the $\mathrm{B}_{\mathrm{c} 2}$ value for the $\mathrm{Mg}$ liquid-state synthesis reaction is dependent mainly on boron grain size and less dependent on the density of the unreacted material.

The transport measurements in Figure 14 show that the thermal treatment under high pressure does not affect the critical temperature for $\mathrm{MgB}_{2}$ wires with large boron and mixtures of boron after the HIP process. However, the sample with nanoboron with a lower filling density of the unreacted material has a lower $T_{c}$ by approximately $2.5 \mathrm{~K}$ for the solid-state synthesis reaction of $\mathrm{Mg}$ and annealing at high isostatic pressure. This result is due to the - lower amount of superconducting phase, unreacted $\mathrm{B}$ and $\mathrm{Mg}$, or the nonstoichiometric superconducting phase. 


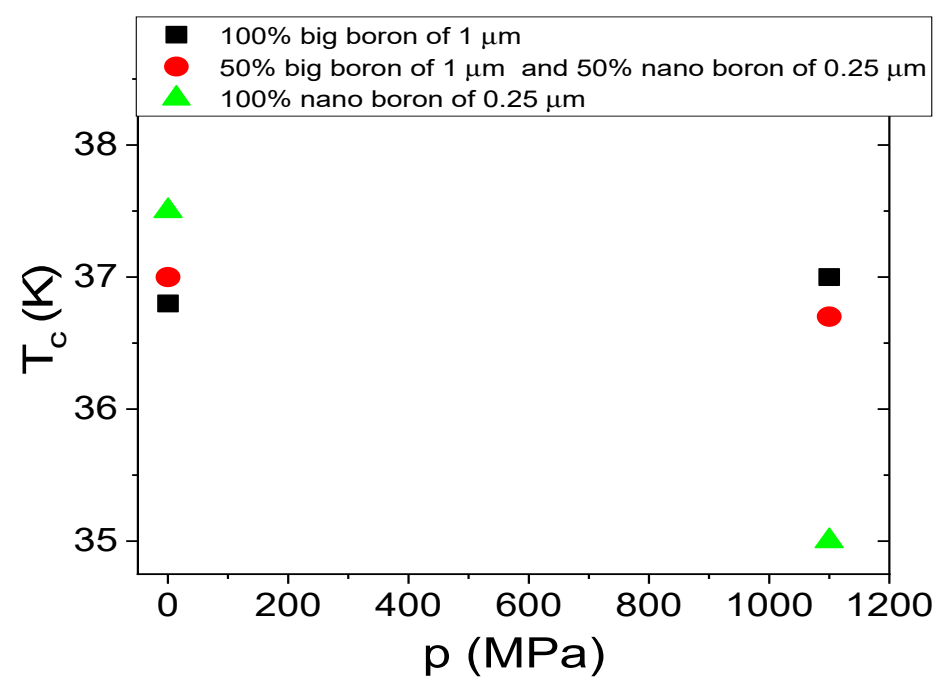

Figure 14. The isostatic pressure $(p)$ dependences of the critical temperature $\left(T_{c}\right)$ for an annealing temperature of $700{ }^{\circ} \mathrm{C}$.

The results in Figure 15 show that increasing the magnetic field from $0 \mathrm{~T}$ to $14 \mathrm{~T}$ leads to a threefold increase in the normal state resistance. Such a large increase in magnetoresistance is caused by the large amount of unreacted $\mathrm{Mg}$ and $\mathrm{B}$, indicating that transport measurements allow for the detection of unreacted particles, e.g., Mg. The transport measurements for samples with large boron grains show that thermal treatment at high isostatic pressure reduces the normal state resistance by approximately $40 \%$. Further studies show that annealing under high isostatic pressure leads to a reduction in the normal state resistance by $50 \%$ in $\mathrm{MgB}_{2}$ wires with the boron grain mixture. Transport measurements for nanoboron grains show that thermal treatment under an isostatic pressure of $1.1 \mathrm{GPa}$ reduces $R_{n}$ by $70 \%$. However, such a large decrease in $R_{n}$ is not only the result of thermal treatment under high isostatic pressure but also of the remnant of unreacted pure $\mathrm{Mg}$, which has a very low resistivity. The reduction of $R_{n}$ is very advantageous because it indicates that the HIP process increases the number of connections between grains.

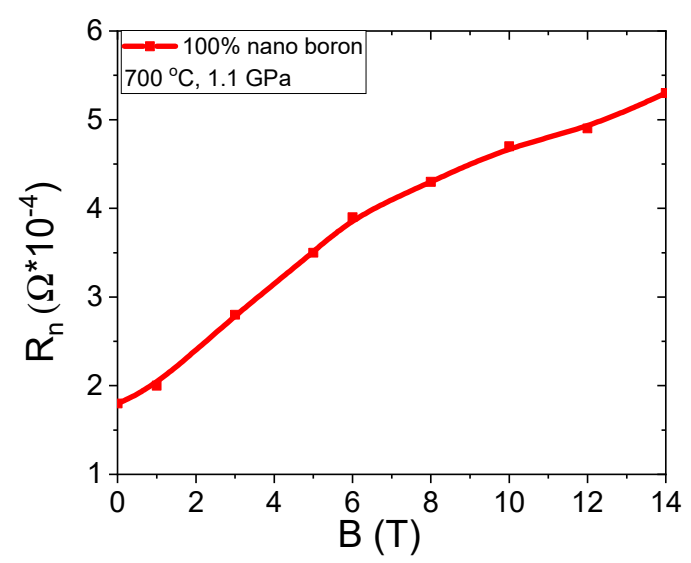

Figure 15. The effect of the magnetic field (B) on the normal state resistance for sample N.

The results in Figure 16 show that thermal treatment under high isostatic pressure increases $\mathrm{Jec}_{\mathrm{ec}}$ by $50 \%$ in $\mathrm{MgB}_{2}$ wires with large boron grains. Transport measurements for $\mathrm{MgB}_{2}$ wires with a boron mixture of grains and thermal treatment under an isostatic pressure of $1.1 \mathrm{GPa}$ increases Jec by $65 \%$ (Figure 16). The SEM photographs (Figures $1 \mathrm{c}, \mathrm{d}$, $2 a, b, 3 c, d$, and $4 a, b$ ) and the results of $B_{\text {irr }}$ (Figure 13a) indicate that $J_{e c}$ is improved with better homogeneity in the structure after the HIP process, e.g., more connections between grains and thinner layered structures. Figure 16 shows that heat treatment under high 
isostatic pressure significantly decreases $\mathrm{Jec}_{\mathrm{ec}}$ by one order of magnitude in $\mathrm{MgB}_{2}$ under medium and high magnetic fields for wires with nanoboron grains, due to the large amount of unreacted $\mathrm{Mg}$ and B material, lower structural homogeneity, and lower filling density of unreacted materials. Studies show that samples with a higher density of unreacted material $(8 \%)$ have a higher $\mathrm{J}_{\mathrm{ec}}$ during the synthesis reaction under high isostatic pressure. However, a sample with unreacted material with a lower filling density after the synthesis reaction under high isostatic pressure has a significantly lower $\mathrm{J}_{\mathrm{ec}}$, despite being made of nanoboron grains, indicating that $\mathrm{J}_{\mathrm{ec}}$ is dependent on the density of the unreacted material and independent of boron grain size during the solid-state $\mathrm{Mg}$ synthesis reaction.

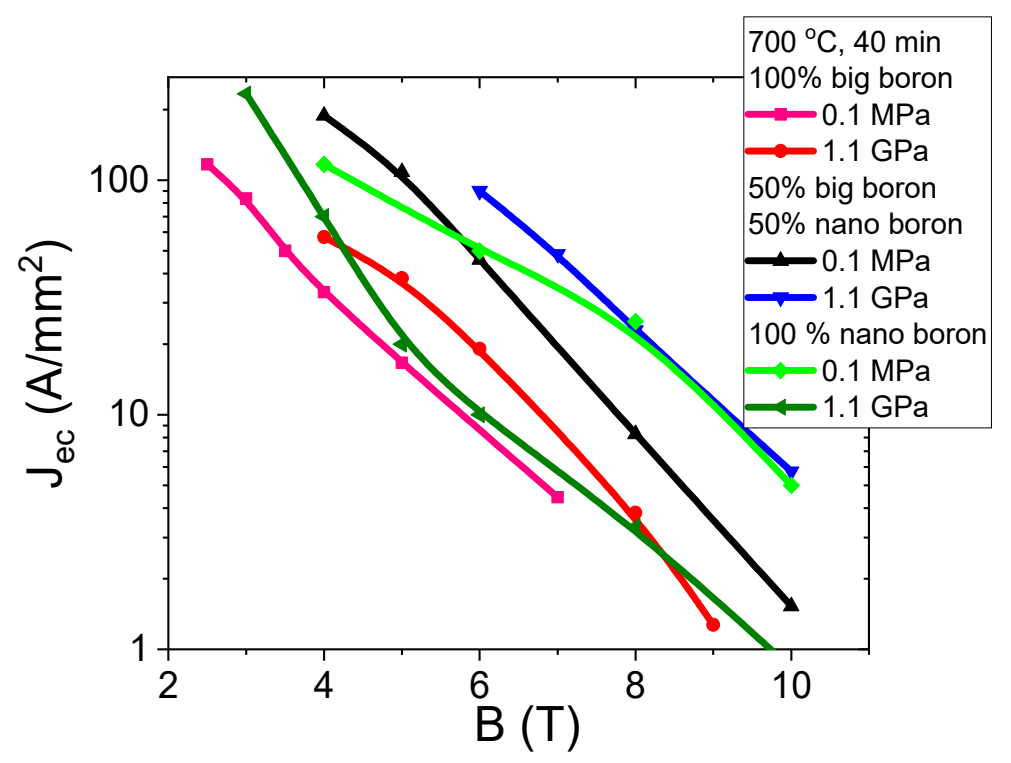

Figure 16. Transport $\mathrm{J}_{\mathrm{ec}}-\mathrm{B}$ curves at $4.2 \mathrm{~K}$ for undoped $\mathrm{MgB}_{2}$ wires in perpendicular magnetic field for annealing temperature of $700{ }^{\circ} \mathrm{C}$.

Figure 17 shows the dependence of the $n$-value on the magnetic field (B) and $\mathrm{Jec}_{\mathrm{ec}}$. These results show that the HIP process and Mg in the solid state weakly increase the $n$-value for $\mathrm{MgB}_{2}$ wires with large boron grains (Figure 17a). The results in Figure 17b show that the heat treatment under high isostatic pressure and the $\mathrm{Mg}$ solid-state synthesis reaction significantly increase the $n$-value for $\mathrm{MgB}_{2}$ wires with a boron grain mixture, indicating that the nanoboron grains, due to their higher density of unreacted material, and high isostatic pressure lead to an improvement in the $n$-value. The results for the sample with nanoboron grains (Figure 17c) indicate that annealing under an isostatic pressure of 1.1 GPa leads to a significant reduction in the $n$-value under medium and high magnetic fields. Such a significant decrease in the $n$-value is a result of the lower filling density of unreacted material in sample $\mathrm{N}$ for the $\mathrm{Mg}$ solid-state reaction. The results in Figure 17 indicate that the $n$-value is dependent mainly on the density of unreacted material and less dependent on the grain size for the solid-state $\mathrm{Mg}$ synthesis reaction. The research presented by Wan et al. [46] shows that $2 \%$ carbon-doped $\mathrm{MgB}_{2}$ PIT wires with nanoboron grains and an $\mathrm{Nb}$ barrier after pressure treatment have an $n$-value of 50 at $7 \mathrm{~T}$. We obtain an $n$-value of 50 at $6.5 \mathrm{~T}$ for undoped $\mathrm{MgB}_{2}$ wires with boron mixture grains $(1 \mu \mathrm{m}$ and $0.25 \mathrm{~nm}$ ) under high isostatic pressure annealing, indicating that our undoped $\mathrm{MgB}_{2}$ wires have high $n$-values and $\mathrm{J}_{\mathrm{ec}}$. Our research showed that the density of unreacted material during the $\mathrm{Mg}$ solid-state synthesis reaction significantly influences the critical parameters $\left(\mathrm{T}_{\mathrm{c}}, \mathrm{B}_{\mathrm{irr}}, \mathrm{B}_{\mathrm{c} 2}, \mathrm{~J}_{\mathrm{ec}}\right.$, and $n$-value). Moreover, we observed that the HIP process improved the homogeneity of the $\mathrm{MgB}_{2}$ material structure in all samples (large boron and boron mixture), which led to a significant improvement in the $\mathrm{Jec}_{\mathrm{ec}}$ and $n$ values. 
(a) boron grain size of $1 \mu \mathrm{m}$

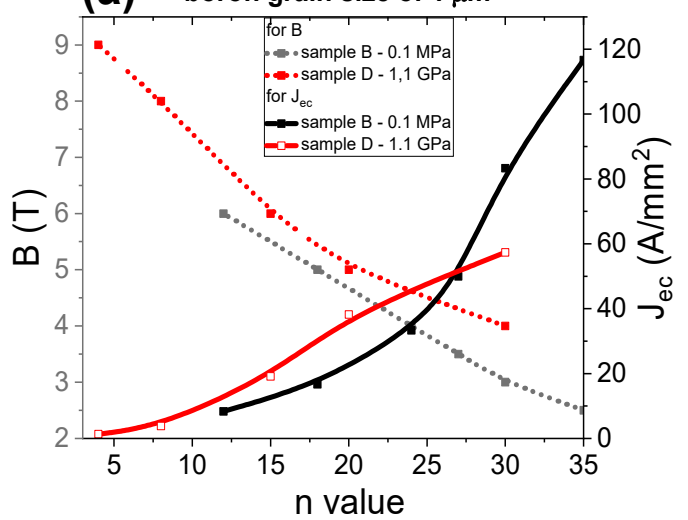

(b) boron grain size of $1 \mu \mathrm{m}$ and $0.25 \mu \mathrm{m}$

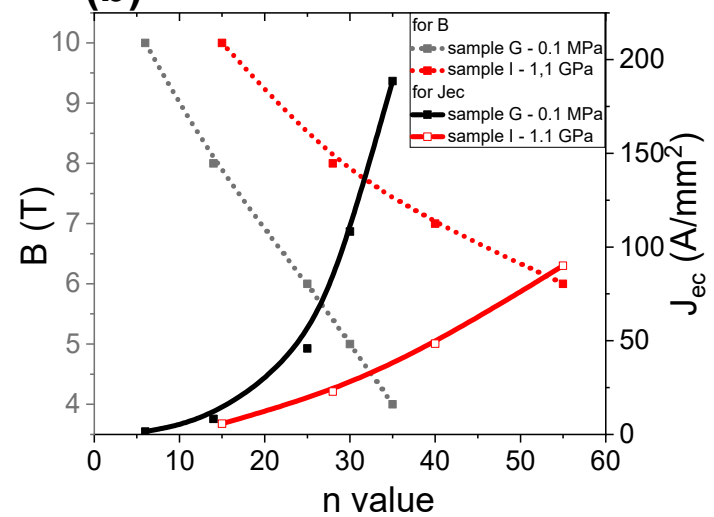

(C) boron grain size of $0.25 \mu \mathrm{m}$

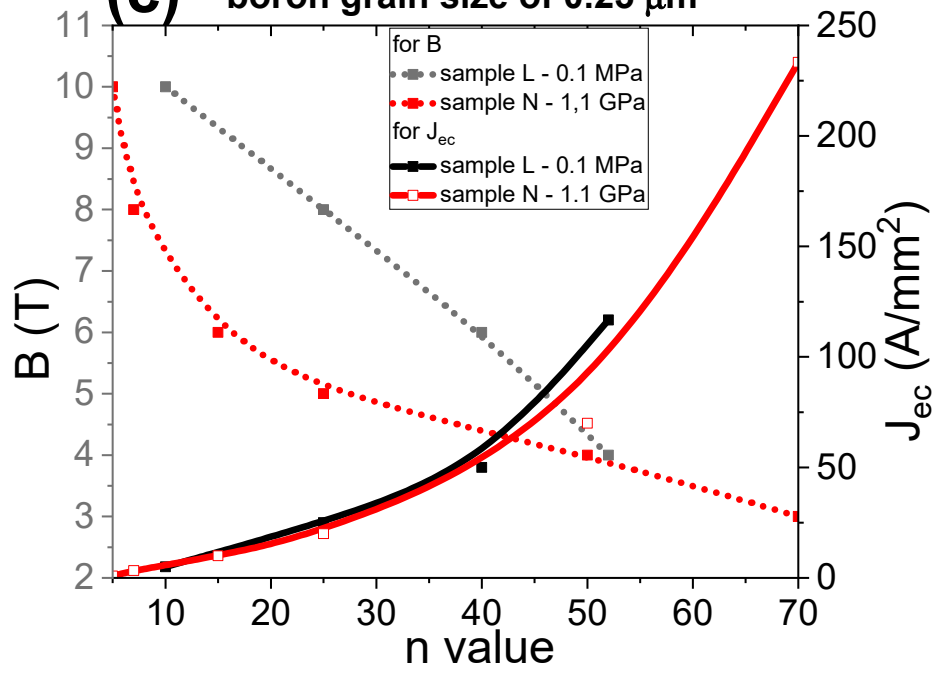

Figure 17. The dependence of the $n$-value on the magnetic field (B) perpendicular to the wire axis in undoped $\mathrm{MgB}_{2}$ wires for an annealing temperature of $700^{\circ} \mathrm{C}$ : (a) boron grain size of $1 \mu \mathrm{m},(\mathbf{b})$ boron grain mixture of $1 \mu \mathrm{m}$ and $0.25 \mu \mathrm{m}$, and (c) boron grain size of $0.25 \mu \mathrm{m}$.

\subsubsection{Thermal Treatment at $740{ }^{\circ} \mathrm{C}$ under High Isostatic Pressure}

The results in [9] show that $\mathrm{Mg}$ is in the liquid state for thermal treatment under an isostatic pressure of $1.1 \mathrm{GPa}$ and an annealing temperature of $740{ }^{\circ} \mathrm{C}$. The results in Figure 18a show that high isostatic pressure annealing increases $\mathrm{B}_{\text {irr }}$ by $30 \%$ for $\mathrm{MgB}_{2}$ wires with large boron grains. Furthermore, transport measurements also showed that thermal treatment under high isostatic pressure increases $\mathrm{B}_{\text {irr }}$ by $10 \%$ in $\mathrm{MgB}_{2}$ wires with mixtures of boron grains (Figure 18a). The following measurements show that the HIP process does not increase $B_{i r r}$ in the $\mathrm{MgB}_{2}$ wires with nanoboron grains (Figure 18a), indicating that high isostatic pressure more effectively increases the density of high-field pinning centers in $\mathrm{MgB}_{2}$ wires with large boron grains during the synthesis reaction in which $\mathrm{Mg}$ is in the liquid state. The transport measurements show that the HIP process slightly increases the density of high-field pinning centers for $\mathrm{MgB}_{2}$ wires with nanoboron grains during the synthesis reaction in which $\mathrm{Mg}$ is in the liquid state. The results of sample $\mathrm{N}\left(700^{\circ} \mathrm{C}\right.$ and $1.1 \mathrm{GPa}$-Figure 13a) and the results of sample $\mathrm{O}\left(740^{\circ} \mathrm{C}\right.$ and $1.1 \mathrm{GPa}$-Figure 18a) show that a higher $B_{\text {irr }}$ for a less unreacted material allows us to obtain a synthesis reaction for $\mathrm{Mg}$ in the liquid state, indicating that high-field pinning centers for unreacted material with a lower filling density are efficiently formed during the HIP process when only Mg is in the liquid state. The measurement results in Figures 13a and 18a for the wires with nanoboron show that increasing the annealing temperature from $700{ }^{\circ} \mathrm{C}$ to $740{ }^{\circ} \mathrm{C}$ for $0.1 \mathrm{MPa}$ isostatic 
pressure does not increase $\mathrm{B}_{\text {irr, }}$ indicating that increasing the annealing temperature from $700{ }^{\circ} \mathrm{C}$ to $740{ }^{\circ} \mathrm{C}$ and $0.1 \mathrm{MPa}$ isostatic pressure slightly improves the high-field centers in $\mathrm{MgB}_{2}$ wires with nanoboron grains.

(a)

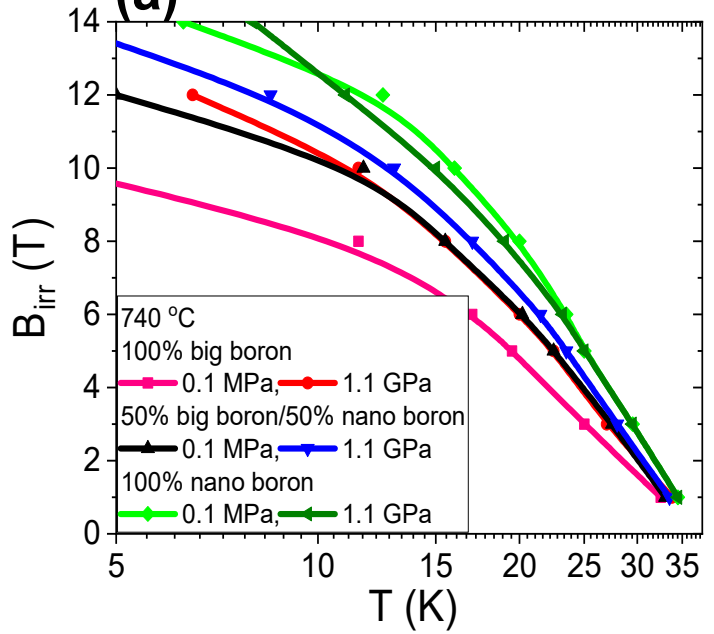

(b)

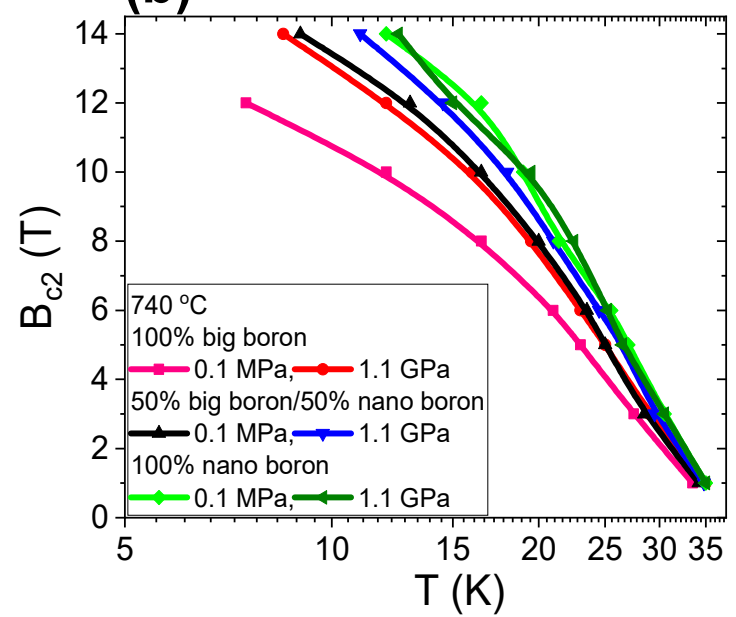

Figure 18. Temperature dependence of the irreversible magnetic field $\left(\mathrm{B}_{\mathrm{irr}}\right)(\mathrm{a})$ and the temperature dependences of the upper magnetic fields $\left(\mathrm{B}_{\mathrm{c} 2}\right)(\mathbf{b})$ for an annealing temperature of $740{ }^{\circ} \mathrm{C}$.

The results in Figure $18 \mathrm{~b}$ show that high-pressure heat treatment at $740{ }^{\circ} \mathrm{C}$ increases $\mathrm{B}_{\mathrm{c} 2}$ by $20 \%$ for $\mathrm{MgB}_{2}$ wires with large boron grains. In Figure $18 \mathrm{~b}$, we see that a high isostatic pressure slightly increases $\mathrm{B}_{\mathrm{c} 2}$ for $\mathrm{MgB}_{2}$ wires with nanoboron grains, indicating that $\mathrm{Mg}$ in the liquid state and the HIP process create a greater number of structural defects, which leads to a greater reduction in the coherence length in $\mathrm{MgB}_{2}$ wires with large boron grains than in $\mathrm{MgB}_{2}$ wires with nanoboron grains. Transport measurements show that the highest $\mathrm{B}_{\mathrm{c} 2}$ is obtained for $\mathrm{MgB}_{2}$ wires with nanoboron grains compared to $\mathrm{MgB}_{2}$ wires with large boron grains, indicating that the nanoboron grains create the largest number of structural defects during the synthesis reaction when $\mathrm{Mg}$ is in the liquid $\mathrm{Mg}$ state. Unreacted material density has little effect on $\mathrm{B}_{\mathrm{c} 2}$ during the synthesis reaction of $\mathrm{Mg}$ in the liquid state.

The transport results in Figure 19 show that the HIP process and the annealing temperature of $740{ }^{\circ} \mathrm{C}$ allow for an increase in the critical temperature $\left(\mathrm{T}_{\mathrm{c}}\right)$ in $\mathrm{MgB}_{2}$ wires with large boron grains and a mixture of boron grains. These results indicate that thermal treatment of $\mathrm{Mg}$ in the liquid state under high isostatic pressure is required for a higher $\mathrm{T}_{\mathrm{c}}$ in $\mathrm{MgB}_{2}$ wires with large boron grains, even for a higher density of unreacted material. A higher $T_{C}$ indicates that a high annealing temperature $\left(740{ }^{\circ} \mathrm{C}\right.$-Figure 19) allows us to obtain a better superconducting phase for wires with large boron grains than a lower thermal treatment temperature under an isostatic pressure of $1.1 \mathrm{GPa}\left(700^{\circ} \mathrm{C}\right.$-Figure 14). The results for samples with nanoboron grains also indicate that thermal treatment at $740{ }^{\circ} \mathrm{C}$ under an isostatic pressure of $1.1 \mathrm{GPa}$ does not improve the $\mathrm{T}_{\mathrm{C}}$ (Figure 19). The results in Figures 14 and 19 for $\mathrm{MgB}_{2}$ wires with nanoboron grains show that the lower filling density of unreacted material requires a higher annealing temperature to obtain a high $\mathrm{T}_{\mathrm{c}}$ during the HIP process. 


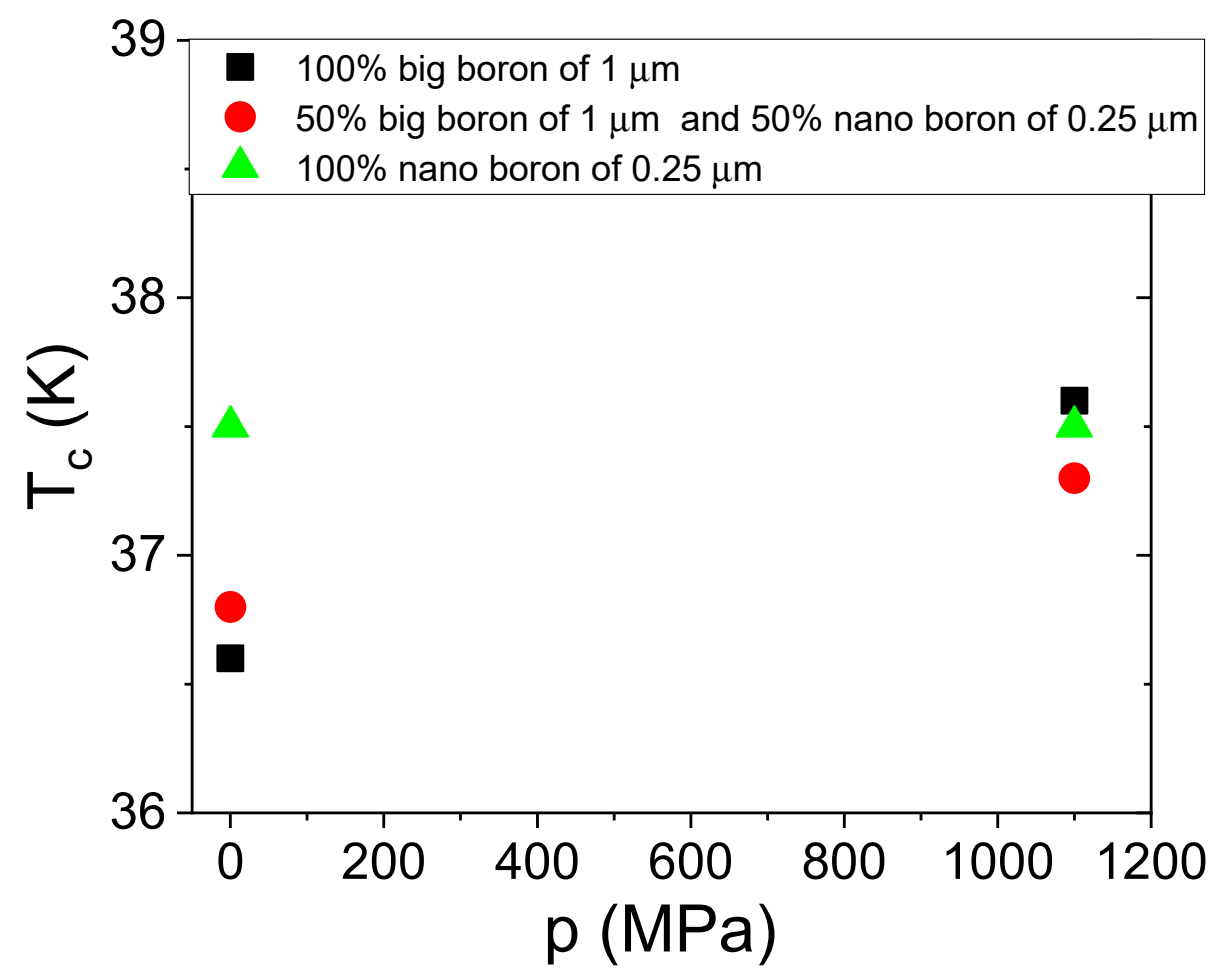

Figure 19. The isostatic pressure $(p)$ dependences of the critical temperature $\left(T_{c}\right)$ for an annealing temperature of $740{ }^{\circ} \mathrm{C}$.

In the previous section, we showed that the magnetoresistance in sample $\mathrm{C}$ is formed by intermetallic phases, e.g., $\mathrm{Mg}, \mathrm{B}$, and Fe (Figure 20). The results in Figure 20 for sample $\mathrm{O}$ (nanoboron grain) show that increasing the magnetic field from $0 \mathrm{~T}$ to $14 \mathrm{~T}$ leads to an increase in the normal state resistance in the range from $10 \%$ to $15 \%$, indicating that sample $\mathrm{O}$ has a small amount of unreacted $\mathrm{Mg}$ and $\mathrm{B}$. The SEM analysis using the AsB detector (Figure 7d) did not display the presence of any unreacted $\mathrm{Mg}$ in sample $\mathrm{O}$. When the results of the magnetoresistance of samples $\mathrm{N}(66 \%)$ with $\mathrm{O}$ (from $10 \%$ to $15 \%$ ) in Figures 15 and 20 are compared, we can conclude that the unreacted material with a lower filling density requires thermal treatment at a higher temperature under an isostatic pressure of $1.1 \mathrm{GPa}$, leading to a significant reduction in the magnetoresistance in $\mathrm{MgB}_{2}$ wires with nanoboron grains. The transport measurements for the annealing temperature of $740^{\circ} \mathrm{C}$ and increasing isostatic pressure from $0.1 \mathrm{MPa}$ to $1.1 \mathrm{GPa}$ showed that normal state resistance increases (for $\mathrm{B}=0 \mathrm{~T}$ ) by $12 \%$ for $\mathrm{MgB}_{2}$ wires with large boron grains and by $6 \%$ for $\mathrm{MgB}_{2}$ wires with nanoboron grains, indicating that thermal treatment in the liquid state of $\mathrm{Mg}$ leads to a reduction in the connections between the grains. Measurements of $\mathrm{MgB}_{2}$ wires with the mixture of boron grains show that the high isostatic pressure and the annealing temperature of $740{ }^{\circ} \mathrm{C}$ reduce $R_{n}$ by $9 \%$, indicating that the mixture of boron grains allows for more intergranular connections. Figure $4 \mathrm{c}$ shows that sample $\mathrm{J}$ has the highest number of connections between grains among all samples. However, the research $\left(740{ }^{\circ} \mathrm{C}\right.$ and $1.1 \mathrm{GPa}$ ) shows that the sample with nanoboron grains has the lowest $\mathrm{R}_{\mathrm{n}}$ value by approximately $25 \%$ compared to the $R_{n}$ for the samples with large grains and a mixture of boron grains, showing that nanoboron grains allow for the highest number of connections between grains, even for the lower filling density of unreacted material during the synthesis reaction in the liquid $\mathrm{Mg}$ state. 


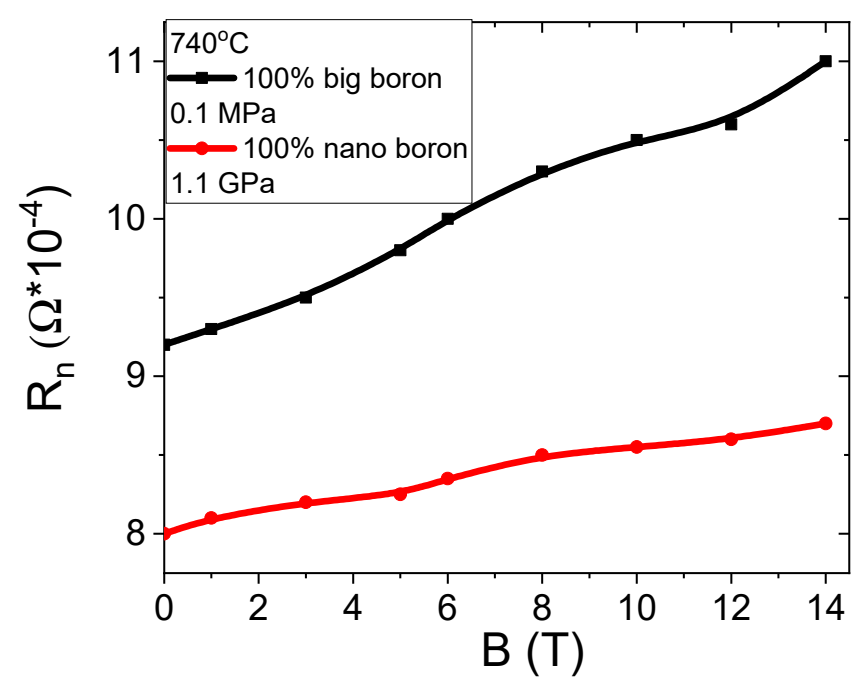

Figure 20. The magnetic field (B) dependences of normal state resistance for samples $\mathrm{C}$ and $\mathrm{N}$.

The results in Figure 21 show that an annealing temperature of $740{ }^{\circ} \mathrm{C}$ and an isostatic pressure of $1.1 \mathrm{GPa}$ increases $\mathrm{Jec}_{\mathrm{ec}}$ by one order of magnitude in $\mathrm{MgB}_{2}$ wires with large boron grains. Transport measurements show that thermal treatment at $740{ }^{\circ} \mathrm{C}$ under an isostatic pressure of $1.1 \mathrm{GPa}$ improves $\mathrm{J}_{\mathrm{ce}}$ under medium and high magnetic fields in $\mathrm{MgB}_{2}$ wires containing mixtures of boron grains (Figure 21). Additional measurements show that a high annealing temperature and high isostatic pressure increase $\mathrm{Jec}_{\mathrm{ec}}$ by $70 \%$ under high magnetic fields in $\mathrm{MgB}_{2}$ wires with nanoboron grains. Our measurements also show that an annealing temperature of $740{ }^{\circ} \mathrm{C}$ and an isostatic pressure of $1.1 \mathrm{GPa}$ lead to a reduction in $\mathrm{Jec}_{\mathrm{ec}}$ by $35 \%$ under medium magnetic fields in $\mathrm{MgB}_{2}$ wires with nanoboron grains. The results in Figure 21 indicate that high isostatic pressure most effectively increases the density of high-field pinning centers in $\mathrm{MgB}_{2}$ wires with nanoboron grains for the synthesis reaction for $\mathrm{Mg}$ in the liquid state. Moreover, the results in Figure 21 indicate that an annealing temperature of $740{ }^{\circ} \mathrm{C}$ and a low isostatic pressure most effectively increase the density of medium field pinning centers in $\mathrm{MgB}_{2}$ wires with nanoboron grains. The longitudinal section of the wires represented in Figures 2c, 4c, and 7a show that thermal treatment at $740{ }^{\circ} \mathrm{C}$ and an isostatic pressure of $1.1 \mathrm{GPa}$ increases the number of connections between grains and allows for the formation of a more homogeneous structure, leading to the improvement of $\mathrm{Jec}_{\mathrm{ec}}$ in $\mathrm{MgB}_{2}$ wires. When the results for the $\mathrm{O}$ sample (nanoboron-740 ${ }^{\circ} \mathrm{C}$ and $1.1 \mathrm{GPa}$ ) are compared to the results for undoped $\mathrm{MgB}_{2}$ wires [43,44], the HIP process is seen to significantly increase $\mathrm{J}_{\mathrm{ec}}$ in high magnetic fields.

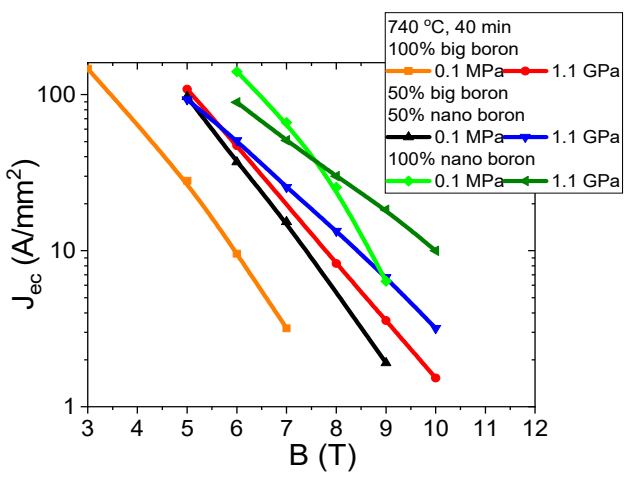

Figure 21. Transport $\mathrm{Jec}_{\mathrm{ec}}-\mathrm{B}$ curves at $4.2 \mathrm{~K}$ for undoped $\mathrm{MgB}_{2}$ wires in perpendicular magnetic fields for an annealing temperature of $740{ }^{\circ} \mathrm{C}$.

The results in Figure 22a show that heat treatment under high isostatic pressure significantly increases the $n$-value in $\mathrm{MgB}_{2}$ wires with large boron grains. The results in 
Figure 22a and [27] suggest that $\mathrm{MgB}_{2}$ wires with large grain boron after the HIP process can be used to build a medium field superconducting coil, e.g., $\mathrm{B}=5 \mathrm{~T}$. These results reveal that the $\mathrm{Mg}$ liquid state and an isostatic pressure of $1.1 \mathrm{GPa}$ significantly improve the $n$-value in $\mathrm{MgB}_{2}$ wires with large boron grains. The higher $n$-value for sample $\mathrm{E}$ is also related to the more homogeneous structure of the $\mathrm{MgB}_{2}$ material after the HIP process (Figures 1e and 2c). Further studies show that the HIP process and an annealing temperature of $740{ }^{\circ} \mathrm{C}$ lead to a significant improvement in the $n$-value under low, medium, and high magnetic fields in $\mathrm{MgB}_{2}$ wires with a mixture of boron grains (Figure 22b). The higher $n$-value for sample $\mathrm{J}$ is also related to the more homogeneous structure of the $\mathrm{MgB}_{2}$ material after the HIP process (Figures $3 e$ and $4 \mathrm{c}$ ). The research in Figure 22c shows that thermal treatment under high isostatic pressure significantly increases the $n$-value, especially for high magnetic fields in $\mathrm{MgB}_{2}$ wires with nanoboron grains. The results in Figure 22 show that the nanoboron grains most efficiently increase the $n$-value under high magnetic fields. Figure 22 shows that the HIP process and an annealing temperature of $740{ }^{\circ} \mathrm{C}$ allow for a high $n$-value under low and medium magnetic fields in $\mathrm{MgB}_{2}$ wires with a mixture of boron grains. The results for sample $\mathrm{O}$ and the results in [46] (undoped $\mathrm{MgB}_{2}$ wires-CTFF method) indicate that the HIP process significantly increases the $n$-value above $7 \mathrm{~T}$. Our research shows that the size of the boron grains significantly influences the critical parameters for the $\mathrm{MgB}_{2}$ material during the HIP process, as well as the $\mathrm{Mg}$ in the liquid state. Additionally, we proved that the HIP process and $\mathrm{Mg}$ in the liquid state improved the homogeneity of the $\mathrm{MgB}_{2}$ material structure, leading to improvements in the $\mathrm{J}_{\mathrm{ec}}$ and $n$-value.

(a) boron grain size of $1 \mu \mathrm{m}$

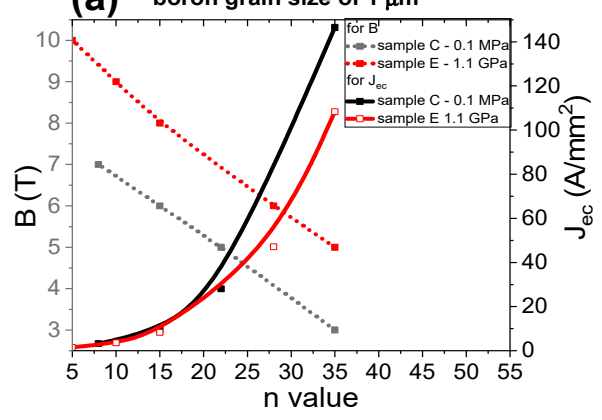

(b) boron grains of $1 \mu \mathrm{m}$ and $0.25 \mu \mathrm{m}$

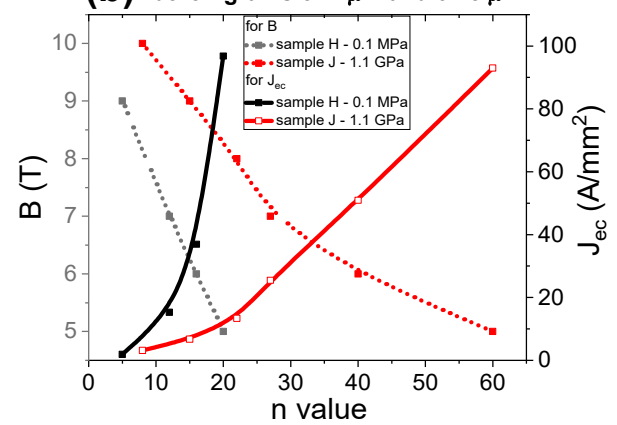

(C) boron grains size of $0.25 \mu \mathrm{m}$

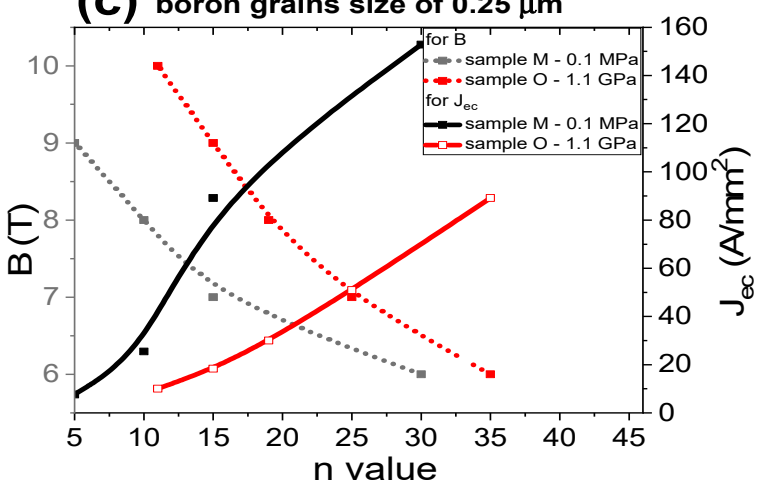

Figure 22. The dependence of the $n$ value on the magnetic field (B) perpendicular to the wire axis in undoped $\mathrm{MgB}_{2}$ wires for an annealing temperature of $740{ }^{\circ} \mathrm{C}$ : (a) boron grain size of $1 \mu \mathrm{m}$, (b) boron grain mixture of $1 \mu \mathrm{m}$ and $0.25 \mu \mathrm{m}$, and (c) boron grain size of $0.25 \mu \mathrm{m}$.

\section{Conclusions}

The results show that a lower filling density of unreacted $\mathrm{Mg}+\mathrm{B}$ material and the $\mathrm{Mg}$ solid-state synthesis reaction lead to a significantly reduced amount of the superconducting phase, a decrease in the homogeneity of the superconducting material, and a 
significant decrease in the irreversible magnetic field, critical temperature, upper magnetic field, engineered critical current density, and $n$-value, despite the high isostatic pressure treatment and the use of nanoboron in the sample. Our measurements show that samples with large boron grains and an $8 \%$ higher filling density of unreacted $\mathrm{Mg}+\mathrm{B}$ material allow to obtain better critical parameters than $\mathrm{MgB}_{2}$ wires with nano boron and a lower filling density of unreacted $\mathrm{Mg}+\mathrm{B}$ material. Based on these results, we can conclude that the density of the unreacted $\mathrm{Mg}+\mathrm{B}$ material has a greater influence on $\mathrm{Mg}$ solid-state synthesis reactions than boron grain size. The experiments conducted indicate that the density of unreacted material has a negligible impact on $B_{i r r}, T_{c}, B_{c 2}, J_{e c}$, and the $n$-value for the $\mathrm{Mg}$ liquid-state synthesis reaction. In addition, studies show that critical parameters $\left(\mathrm{T}_{\mathrm{c}}, \mathrm{B}_{\mathrm{irr}}, \mathrm{B}_{\mathrm{c} 2}, \mathrm{~J}_{\mathrm{ec}}\right.$, and $n$-value) of $\mathrm{MgB}_{2}$ wires depend mainly on the boron grain size for the $\mathrm{Mg}$ liquid-state synthesis reaction. Nanoboron grains and the HIP process allow for the highest $\mathrm{B}_{\text {irr }}, \mathrm{T}_{\mathrm{c}}, \mathrm{B}_{\mathrm{c} 2}, \mathrm{~J}_{\mathrm{ec}}$, and $n$-value under high magnetic fields. The SEM images taken from the longitudinal section of the wires show that the samples annealed under low isostatic pressure have a highly heterogeneous structure. The HIP process significantly increases the homogeneity of the $\mathrm{MgB}_{2}$ material. Transport measurements show that an isostatic pressure of $1.1 \mathrm{GPa}$ and an annealing temperature of $740^{\circ} \mathrm{C}$ allow for the highest $\mathrm{J}_{\text {ec }}$ and $n$-value for $\mathrm{MgB}_{2}$ wires with large boron. In addition, the experiments show that the nano boron grains and the solid-state reaction of $\mathrm{Mg}$ allow for the formation of the highest number of high-field pinning centers. However, thermal treatment in the liquid state of $\mathrm{Mg}$ creates a large number of low- and medium-field pinning centers in $\mathrm{MgB}_{2}$ wires with nanoboron. Research has shown that the $n$-value is most decreased in the presence of island structures. The $n$-value has been found to be the best in structures with thin layers and homogeneous layer distributions. The results have shown that layer clustering also results in a reduction in the $n$-value.

Author Contributions: Conceptualization, D.G., A.J.Z. and T.C.; methodology, A.M. and I.B.; formal analysis, D.G., A.J.Z., I.B., T.C.; investigation, D.G., A.M., M.M., M.A., F.K., D.A. and H.Y.; resources, M.A., H.Y., I.B.; writing-original draft preparation, D.G.; writing—review and editing, A.J.Z.; visualization, T.C. All authors have read and agreed to the published version of the manuscript.

Funding: This research was funded by the Institute of Low Temperature and Structure Research, PAS, the Institute of High Pressure Physics, PAS and Ministry of National Defense Republic of Poland Program-Research Grant MUT Project 13-995. The wire samples of this work were fabricated with financial support from the Scientific and Technological Research Council of Turkey (TUBITAK) research project with grant numbers 217 M665.

Institutional Review Board Statement: Not applicable.

Informed Consent Statement: Not applicable.

Data Availability Statement: The data presented in this study are available on reasonable request from the corresponding authors.

Conflicts of Interest: The authors declare no conflict of interest.

\section{References}

1. Kim, J.H.; Heo, Y.U.; Matsumoto, A.; Kumakura, H.; Rindfeisch, M.; Tomsic, M.; Dou, S.X. Comparative study of mono-and multi-filament $\mathrm{MgB}_{2}$ wires with different boron powders and malic acid addition. Supercond. Sci. Technol. 2010, $23,075014$. [CrossRef]

2. Ballarino, A.; Flükiger, R. Status of $\mathrm{MgB}_{2}$ wire and cable applications in Europe. J. Phys. Conf. Ser. 2017, 871, $012098-0120105$. [CrossRef]

3. Zhang, Y.; Xu, X.; Zhan, Y.; Kim, J.H.; Lu, C.; Zhou, S.H.; Dou, S.X. Significant improvement of $\mathrm{J}_{\mathrm{C}}$ in $\mathrm{MgB}_{2}$ bulk superconductor using ball-milled high-purity crystalline boron. Supercond. Sci. Technol. 2008, 21, 115004-115010. [CrossRef]

4. Chen, S.K.; MacManus-Driscoll, J.L. Enhanced critical current densities in $\mathrm{MgB}_{2}$ by mixing relatively impure boron powders. Supercond. Sci. Technol. 2009, 22, 065009-065014. [CrossRef]

5. Xu, D.; Wang, D.; Li, C.; Yuan, P.; Zhang, X.; Yao, C.; Dong, C.; Huang, H.; Ma, Y.; Oguro, H.; et al. Microstructure and superconducting properties of nanocarbon-doped internal $\mathrm{Mg}$ diffusion-processed $\mathrm{MgB}_{2}$ wires fabricated using different boron powders. Supercond. Sci. Technol. 2016, 29, 045009-045019. [CrossRef] 
6. Gajda, D.; Morawski, A.; Zaleski, A.J.; Akdogan, M.; Yetis, H.; Karaboga, F.; Cetner, T.; Belenli, I. The influence of HIP process on critical parameters of $\mathrm{MgB}_{2} / \mathrm{Fe}$ wires with big boron grains and without barriers. J. Alloy. Compd. 2016, 687, 616-622. [CrossRef]

7. Bovone, G.; Matera, D.; Bernini, C.; Magi, E.; Vignolo, M. Manufacturing process influence on superconducting properties of $\mathrm{MgB}_{2}$ wires prepared using laboratory made boron. Supercond. Sci. Technol. 2015, 28, 065006-065016. [CrossRef]

8. Serquis, A.; Civale, L.; Hammon, D.L.; Liao, X.Z.; Coulter, J.Y.; Zhu, Y.T.; Jaime, M.; Peterson, D.E.; Mueller, F.M. Hot isostatic pressing of powder in tube $\mathrm{MgB}_{2}$ wires. Appl. Phys. Lett. 2003, 82, 2847-2849. [CrossRef]

9. Gajda, D.; Morawski, A.; Zaleski, A.J.; Cetner, T.; Häßler, W.; Nenkov, K.; Małecka, M.; Rindfleisch, M.A.; Tomsic, M. Significant enhancement of the critical current of $\mathrm{MgB}_{2}$ wires through a reduction of the diameter using HIP method. Scr. Mater. 2018, 143, 77-80. [CrossRef]

10. Cetner, T.; Morawski, A.; Adamczyk, K.; Rindfleisch, M.; Tomsic, M.; Zaleski, A.J.; Gajda, D.; Presz, A. Improvement of critical properties of undoped, multifilamentary $\mathrm{MgB}_{2}$ wires in $\mathrm{Nb} / \mathrm{Cu}$ after annealing under high gas pressure. High. Press. Res. 2012, 32, 419-424. [CrossRef]

11. Pélissier, J.L. Determination of the phase diagram of magnesium: A model-potential approach in the sub-megabar range. Phys. Scr. 1986, 34, 838-841. [CrossRef]

12. Avedesian, M.M.; Baker, H. Magnesium and Magnesium Alloys; ASM International: Materials Park, OH, USA, $1999 ;$ pp. 7-11.

13. Jie, H.; Qiu, W.; Billah, M.; Mustapic, M.; Patel, D.; Ma, Z.; Gajda, D.; Morawski, A.; Cetner, T.; Shahabuddin, M.; et al. Superior transport $\mathrm{J}_{\mathrm{C}}$ obtained in in-situ $\mathrm{MgB}_{2}$ wires by tailoring the starting materials and using a combined cold high pressure densification and hot isostatic pressure treatment. Scr. Mater. 2017, 129, 79-83. [CrossRef]

14. Adamczyk, K.; Morawski, A.; Cetner, T.; Zaleski, A.J.; Gajda, D.; Rindfleisch, M.; Tomsic, M.; Diduszko, R.; Presz, A. Superconducting properties comparison of $\mathrm{SiC}$ doped multifilamentary $\mathrm{MgB}_{2}$ wires of various sheaths $(\mathrm{Cu}$, Monel, Glidcop) after high pressure HIP treatment. IEEE Trans. Appl. Supercond. 2012, 22, 6200204-6200209. [CrossRef]

15. Li, C.; Suo, H.; Ma, L.; Yang, L.; Meada, M.; Patel, D.; Matsumoto, A.; Kumakura, H. Significant improvement in superconducting properties of in situ powder-in-tube $\mathrm{MgB}_{2}$ wires through anthracene doping and heat treatment optimization. Supercond. Sci. Technol. 2019, 32, 105004-105013. [CrossRef]

16. Flükiger, R.; Suo, H.L.; Musolino, N.; Beneduce, C.; Toulemonde, P.; Lezza, P. Superconducting properties of $\mathrm{MgB}_{2}$ tapes and wires. Phys. C Supercond. 2003, 385, 286-305. [CrossRef]

17. Mackinnon, I.; Shabazi, M.; Alarco, J.; Talbot, P.C. Low temperature decomposition of metal borohydride drives autogenous synthesis of $\mathrm{MgB}_{2}$. Supercond. Sci. Technol. 2017, 30, 055004-055018. [CrossRef]

18. Mroczek, Z.; Morawski, A.; Czujko, T.; Karaboğa, F.; Akdoğan, M.; Zaleski, A.J.; Małecka, M.; Cetner, T.; Yetiş, H.; Gajda, D.; et al. Influence of the lamella structure and high isostatic pressure on the critical current density in in situ $\mathrm{MgB}_{2}$ wires without a barrier. J. Alloy. Compd. 2019, 776, 636-645. [CrossRef]

19. Flükiger, R.; Hossain, M.S.A.; Senatore, C. Strong enhancement of $\mathrm{J}_{\mathrm{C}}$ in binary and alloyed in-situ $\mathrm{MgB}_{2}$ wires by a new approach: Cold high pressure densification. Supercond. Sci. Technol. 2009, 22, 085002-085009. [CrossRef]

20. Hossain, M.S.A.; Senatore, C.; Rindfleisch, M.; Flükiger, R. Improvement of $J_{c}$ by cold high pressure densification of binary, 18-filament in situ $\mathrm{MgB}_{2}$ wires. Supercond. Sci. Technol. 2011, 24, 075013-075018. [CrossRef]

21. Akdoğan, M.; Yetiş, H.; Gajda, D.; Karaboğa, F.; Ülgen, A.T.; Demirtürk, E.; Belenli, İ. Effect of the initial filling density on the critical current of in-situ Fe/MgB 2 wires. J. Alloy. Compd. 2015, 649, 1007-1010. [CrossRef]

22. Zhou, S.; Pan, A.V.; Liu, H.; Dou, S. Single-and multi-filamentary Fe-sheathed $\mathrm{MgB}_{2}$ wires. Phys. C Supercond. 2002, 382, 349-354. [CrossRef]

23. Tanaka, K.; Okada, M.; Kumakura, H.; Kitaguchi, H.; Togano, K. Fabrication and transport properties of $\mathrm{MgB}_{2}$ wire and coil. Phys. C Supercond. 2002, 382, 203-206. [CrossRef]

24. Susner, M.A.; Sumption, M.D.; Bhatia, M.; Tomsic, M.J.; Rindfleisch, M.A.; Collings, E.W. Changes in microstructure and transport properties of magnesium diboride strands through hot uniaxial pressing. AIP Conf. Proc. 2008, 986, 375-381.

25. Kim, J.H.; Dou, S.X.; Matsumoto, A.; Choi, S.; Kiyoshi, T.; Kumakura, H. Correlation between critical current density and $n$-value in $\mathrm{MgB}_{2} / \mathrm{Nb} /$ Monel superconductor wires. Phys. C Supercond. Appl. 2010, 470, 1207-1210. [CrossRef]

26. Motaman, A.; Barua, S.; Patel, D.; Maeda, M.; Cheong, K.; Kim, J.H.; Dou, S.X.; Hossain, M.S.A. Power-law relationship between critical current density, microstructure, and the $n$-value in $\mathrm{MgB}_{2}$ superconductor wires. J. Supercond. Nov. Magn. 2014, 27, 1643-1645. [CrossRef]

27. Parizh, M.; Lvovsky, Y.; Sumption, M. Conductors for commercial MRI magnets beyond NbTi: Requirements and challenges. Supercond. Sci. Technol. 2017, 30, 014007. [CrossRef] [PubMed]

28. Rogalla, H.; Kes, P.H. 100 Years of Superconductivity; Chapman and Hall: London, UK, 2011; pp. 713-826.

29. Gajda, D.; Zaleski, A.J.; Morawski, A.; Haßler, W.; Nenkov, K.; Rindfleisch, M.A.; Hossain, M.S.A. Pinning mechanism and engineering critical current density considerations in the design of $\mathrm{MgB}_{2}$ superconducting coils. Phys. C Supercond. Appl. 2020, 570, 1353606-1353624. [CrossRef]

30. Rahul, S.; Devadas, K.M.; Thomas, S.; Varghese, N.; Ajeesh, P.; Paulose, M.; Manoj, R.V.; Syamaprasad, U. A comparative study on the effects of $\mathrm{n} \mathrm{C}, \mathrm{n} \mathrm{SiC}$ and $\mathrm{BRH}$ on the structural and superconducting properties of $\mathrm{MgB}_{2}$ PIT wires. Mater. Chem. Phys. 2017, 200, 395-401. [CrossRef] 
31. Xiang, F.X.; Wang, X.L.; Xun, X.; De Silva, K.S.; Wang, Y.X.; Dou, S.X. Evidence for transformation from $\delta \mathrm{T}_{\mathrm{C}}$ to $\delta 1$ pinning in $\mathrm{MgB}_{2}$ by graphene oxide doping with improved low and high field $\mathrm{J}_{\mathrm{c}}$ and pinning potential. Appl. Phys. Lett. 2013, 102, 152601-152606. [CrossRef]

32. Yamada, H.; Igarashi, M.; Nemoto, Y.; Yamada, Y.; Tachikawa, K.; Kitaguchi, H.; Matsumoto, A.; Kumakura, H. Influence of heat treatment on in situ powder-in-tube-processed $\mathrm{MgB}_{2}$ tapes with added ethyltoluene and SiC powder. Supercond. Sci. Technol. 2009, 22, 075005-075010. [CrossRef]

33. Nagamatsu, J.; Nakagawa, N.; Muranaka, T.; Zenitan, Y.; Akimitsu, J. Superconductivity at 39 K in magnesium diboride. Nature 2001, 410, 63-64. [CrossRef]

34. Canfield, P.C.; Finnemore, D.K.; Budko, S.L.; Ostenson, J.E.; Lapertot, G.; Cunningham, C.E.; Petrovi, C. Superconductivity in dense $\mathrm{MgB}_{2}$ wires. Phys. Rev. Lett. 2001, 86, 2423-2426. [CrossRef]

35. Gajda, D.; Morawski, A.; Zaleski, A.J.; Akdoğan, M.; Yetiş, H.; Karaboğa, F.; Cetner, T.; Belenli, I. Formation of high-field pinning centers in superconducting $\mathrm{MgB}_{2}$ wires by using high hot isostatic pressure process. J. Supercond. Nov. Magn. 2017, 30, 3397-3402. [CrossRef]

36. Blatter, G.; Feigel'man, M.V.; Geshkenbein, V.B.; Larkin, A.I.; Vinokur, V.M. Vortices in high-temperature superconductors. Rev. Mod. Phys. 1994, 66, 1125-1388. [CrossRef]

37. Gajda, D.; Zaleski, A.J.; Morawski, A.; Hossain, M.S.A. New types of high field pinning centers and pinning centers for the peak effect. Supercond. Sci. Technol. 2017, 30, 085011-085020. [CrossRef]

38. Kazakov, S.M.; Puzniak, R.; Rogacki, K.; Mironov, A.V.; Zhigadlo, N.D.; Jun, J.; Soltmann, C.; Batlogg, B.; Karpinski, J. Carbon substitution in $\mathrm{MgB}_{2}$ single crystals: Structural and superconducting properties. Phys. Rev. B 2005, 71, 024533-024543. [CrossRef]

39. Dou, S.X.; Shcherbakova, O.; Yeoh, W.K.; Kim, J.H.; Soltanian, S.; Wang, X.L.; Senatore, C.; Flükiger, R.; Dhallé, M.; Husnjak, O.; et al. Mechanism of Enhancement in Electromagnetic Properties of $\mathrm{MgB}_{2}$ by Nano SiC Doping. Phys. Rev. Lett. 2007, 98, 097002-097006. [CrossRef]

40. Buzea, C.; Yamashita, T. Review of the superconducting properties of MgB2. Supercond. Sci. Technol. 2001, 14, R115-R146. [CrossRef]

41. Li, G.Z.; Sumption, M.D.; Zwayer, J.B.; Susner, M.A.; Rindfeisch, M.A.; Thong, C.J.; Tomsic, M.J.; Colling, E.W. Effects of carbon concentration and filament number on advanced internal $\mathrm{Mg}$ infiltration-processed $\mathrm{MgB}_{2}$ strands. Supercond. Sci. Technol. 2013, 26, 095007-095016. [CrossRef]

42. Li, G.Z.; Sumption, M.D.; Susner, M.A.; Yang, Y.; Reddy, K.M.; Rindfeisch, M.A.; Tomsic, M.J.; Thong, C.J.; Colling, E.W. The critical current density of advanced internal-Mg-diffusion-processed MgB 2 wires. Supercond. Sci. Technol. 2012, 25, 115023-115031. [CrossRef]

43. Li, G.Z.; Yang, Y.; Susner, M.A.; Sumption, M.D.; Colling, E.W. Critical current densities and $n$-values of MgB 2 strands over a wide range of temperatures and fields. Supercond. Sci. Technol. 2012, 25, 025001-025011. [CrossRef]

44. Ye, S.J.; Matsumoto, A.; Togano, K.; Kumakura, H. Enhancement of the critical current density of internal Mg diffusion processed $\mathrm{MgB}_{2}$ wires by the addition of both $\mathrm{SiC}$ and liquid aromatic hydrocarbon. Phys. C Supercond. Appl. 2011, 471, 1133-1136. [CrossRef]

45. Gajda, D.; Zaleski, A.J.; Morawski, A.; Cetner, T.; Małecka, M.; Rindfleisch, M. The Optimization of J $\mathrm{J}_{\mathrm{C}}, \mathrm{B}_{\text {irr }}, n$ value, wires diameter, and research of the dominant pinning mechanism for applications of undoped $\mathrm{MgB}_{2}$ in superconducting multi-section coils. $J$. Supercond. Nov. Magn. 2020, 33, 3717-3725. [CrossRef]

46. Wan, F.; Sumption, M.D.; Rindfeisch, M.A.; Colling, E.W. Pressure-induced property improvement of magnesium diboride wire. IOP Conf. Ser. Mater. Sci. Eng. 2017, 279, 012024-012031. [CrossRef] 